Plasma rotation and transport in MAST spherical tokamak

This content has been downloaded from IOPscience. Please scroll down to see the full text. 2011 Nucl. Fusion 51063006

(http://iopscience.iop.org/0029-5515/51/6/063006)

View the table of contents for this issue, or go to the journal homepage for more

Download details:

IP Address: 150.203.179.67

This content was downloaded on 04/08/2014 at 06:32

Please note that terms and conditions apply. 


\title{
Plasma rotation and transport in MAST spherical tokamak
}

\author{
A.R. Field ${ }^{1}$, C. Michael ${ }^{1}$, R.J. Akers ${ }^{1}$, J. Candy ${ }^{2}$, G. Colyer ${ }^{1,3}$, \\ W. Guttenfelder ${ }^{4}$, Y.-c. Ghim ${ }^{3}$, C.M. Roach $^{1}$, S. Saarelma ${ }^{1}$ and \\ the MAST Team \\ ${ }^{1}$ EURATOM/CCFE Fusion Association, Culham Science Centre, Abingdon, Oxon, UK \\ ${ }^{2}$ General Atomics, PO Box 85608, San Diego, CA 92186-5608, USA \\ ${ }^{3}$ Rudolf Peierls Centre for Theoretical Physics, University of Oxford, Oxford, UK \\ ${ }^{4}$ Princeton Plasma Physics Laboratory, PO Box 451, Princeton, NJ, USA \\ E-mail: anthony.field@ccfe.ac.uk
}

Received 6 December 2010, accepted for publication 24 March 2011

Published 28 April 2011

Online at stacks.iop.org/NF/51/063006

\begin{abstract}
The formation of internal transport barriers (ITBs) is investigated in MAST spherical tokamak plasmas. The relative importance of equilibrium flow shear and magnetic shear in their formation and evolution is investigated using data from high-resolution kinetic- and $q$-profile diagnostics. In L-mode plasmas, with co-current directed NBI heating, ITBs in the momentum and ion thermal channels form in the negative shear region just inside $q_{\text {min }}$. In the ITB region the anomalous ion thermal transport is suppressed, with ion thermal transport close to the neo-classical level, although the electron transport remains anomalous. Linear stability analysis with the gyro-kinetic code GS2 shows that all electrostatic micro-instabilities are stable in the negative magnetic shear region in the core, both with and without flow shear. Outside the ITB, in the region of positive magnetic shear and relatively weak flow shear, electrostatic micro-instabilities become unstable over a wide range of wave numbers. Flow shear reduces the linear growth rates of low- $k$ modes but suppression of ITG modes is incomplete, which is consistent with the observed anomalous ion transport in this region; however, flow shear has little impact on growth rates of high- $k$, electron-scale modes. With counter-NBI ITBs of greater radial extent form outside $q_{\text {min }}$ due to the broader profile of $E \times B$ flow shear produced by the greater prompt fast-ion loss torque.
\end{abstract}

(Some figures in this article are in colour only in the electronic version)

\section{Introduction}

Shear-flow suppression of anomalous turbulent transport plays an important role in determining the confinement properties of spherical tokamak (ST) plasmas. The low moment of inertia of such plasmas means that high rates of toroidal rotation, and hence equilibrium flow shear, can be driven by the torque from neutral beam injection (NBI) heating. The mega amp spherical tokamak (MAST) is a low aspect ratio, medium sized tokamak ( $A=R / a \sim 1.3$, plasma current $I_{\mathrm{p}} \leqslant 1.3 \mathrm{MA}$, toroidal field $\left.B_{\mathrm{t}} \leqslant 0.57 \mathrm{~T}\right)$, which is equipped with high-power, tangentially directed NBI heating (injected power $P_{\text {inj }} \leqslant 4.2 \mathrm{MW}$, beam energy $E_{0} \leqslant 70 \mathrm{keV}$ ). This heating drives strong toroidal rotation, with typical Mach number $M_{\phi}=R \omega_{\phi} / v_{\text {th, }} \leqslant$ 0.5 (where $\omega_{\phi}$ is the rotation rate and $v_{\text {th,i }}$ the ion thermal velocity). The resulting perpendicular $\left(E_{r} \times B\right)$ shearing rates can be sufficient to exceed the growth rate of the low- $k$ ionscale turbulence and hence suppress the associated anomalous transport in the ion and momentum channels.
In H-mode plasmas in MAST, ion heat transport is within a factor of 1-3 of the ion neo-classical level over most of the plasma radius, while the electron transport remains highly anomalous $[1,2]$ relative to the electron neo-classical level. In L-mode plasmas, however, the ion transport can significantly exceed neo-classical levels in the outer regions but is suppressed by flow shear at mid-radius and under favourable conditions can exhibit an internal transport barrier (ITB) with ion transport at the neo-classical level. The ITB scenario studied here relies on early NBI heating of a low-density plasma applied during the current ramp-up phase. This slows the current penetration, resulting in negative magnetic shear in the core plasma and strong equilibrium flow shear, both of which reduce the level of anomalous transport due to microturbulence and hence favour ITB formation.

Studies of transport in such regimes in MAST are facilitated by the availability of advanced diagnostics, including a high-resolution, multi-pulse NdYAG Thomson scattering system [3] with spatial resolution $(\Delta R \sim 1 \mathrm{~cm})$ 
matching that of the CXRS system [4] and a multichannel motional Stark effect (MSE) diagnostic for $q$-profile measurements [5]. An integrated analysis chain $\left(\mathrm{MC}^{3}\right)$ is used to prepare the input data for transport analysis using TRANSP [6]. This now incorporates EFIT equilibrium reconstructions constrained using the magnetic field pitch angle data from the MSE measurements. Knowledge of the measured $q$-profile evolution consequently allows the local transport properties to be related to the magnetic shear and location of low-order rational surfaces.

A brief overview of mechanisms which have been suggested might initiate ITB formation is presented in section 2. The phenomenology of MAST discharges exhibiting ITBs, heated with both co- and counter-current directed, tangential NBI heating, is presented in section 3, including results of local transport analysis. The location and timing of ITB formation is related to the $q$-profile evolution and the magnetic shear. The role of MHD activity in limiting the ITB strength and in their termination is also discussed. In section 4 results of micro-stability analysis of a co-NBI ITB discharge are presented both from the linear gyro-kinetic flux-tube code GS2, which incorporates flow shear [7], and also with the global, particle in cell (PIC) code ORB5 [8]. These analyses reveal the relative importance of flow shear and magnetic shear to micro-stability and hence to the level of anomalous transport. In particular, that negative magnetic shear in the plasma core is strongly stabilizing for the microinstabilities hence favouring ITB formation. The paper concludes with a summary of findings and conclusions drawn in section 5 .

\section{ITB formation mechanisms}

An ITB can be defined as a region of reduced anomalous transport in the plasma core, associated with strong $E \times B$ flow shear, low or negative magnetic shear $\hat{s}=r / q \cdot(\mathrm{d} q / \mathrm{d} r)$ and a suppression of turbulence $[9,10]$. In several tokamaks ITB formation is observed to be linked to low-order rational surfaces, where the transport improvement is initiated by or tied to locations with rational $q$ values. In many cases the ITB is initiated or strengthened when the location of zero magnetic shear $\left(q_{\min }\right)$ passes through a low-order rational value.

Two classes of mechanism proposed for ITB formation are related to coherent MHD modes and to the density of rational surfaces, respectively. In the former class [11,12], tearing modes or fast-particle driven 'fishbone' modes are thought to modify the radial electric field $\left(E_{r}\right)$ profile and hence trigger the ITB through increased $E \times B$ flow shear suppression of the turbulence. The fishbone modes redistribute the fast ions radially, which results in a $j_{r} \times B$ torque due to the return current in the plasma. This torque modifies the rotation profile and hence $E_{r}$. In the case of the tearing modes, it is suggested in [9] that the profile flattening due to the islands locally modifies the pressure gradients and hence $E_{r}$. The second class of mechanism relies on the rarefaction of low-order rational surfaces in the vicinity of integer $q$ values $[13,14]$, which could lead to decreased interaction between micro-instabilities and hence a local reduction in the anomalous transport. In regions of weak or negative magnetic shear, growth rates of micro-instabilities are also reduced due to a decrease in the interchange drive $[15,16]$, which hence favours shear flow suppression and facilitates ITB formation.

In DIII-D [17], discharges exhibiting transport changes at rational $q$ values are low-density, L-mode discharges with NBI heating power marginal for ITB formation applied early to produce negative magnetic shear in the plasma core. Steepening in gradients of $T_{\mathrm{i}}$ and toroidal rotation $\omega_{\phi}$ is observed to be correlated with times when $q_{\text {min }}$ passes through integer values, with a simultaneous reduction in low- $k$ turbulence observed with the BES diagnostic. On JET [11], ITBs have also been linked to integer $q$ values, forming when $q_{\text {min }}$ reaches an integer value and then bifurcating, propagating both inwards and outwards with foot points following the two integer $q$ surfaces. In NSTX [18] ITBs in the ion thermal and momentum channels are observed to form in the vicinity of maximum $E \times B$ shear, consistent with the $E \times B$ shear stabilization of ITG turbulence, while electron ITBs are more closely correlated with the region of most negative magnetic shear.

\section{ITB discharges}

\subsection{Discharge scenario}

The ITB scenario used on MAST [19,20] is similar to that of other tokamaks, with early NBI heating of a lowdensity, L-mode discharge applied during the current ramp to slow current penetration, resulting in strong toroidal rotation and reversed magnetic shear in the core. One aim is to investigate the relative importance of rotation shear compared with magnetic shear by comparing discharges heated with NBI heating injected either in the co- or counter-current directions. Because the orientation of the beams is fixed, to achieve counter-current injection the usual directions of the plasma current and toroidal field (which are anti-clockwise and clockwise view from above) are both reversed.

With the usual co-current injection, the poloidal field is oriented such that the first orbits of the fast ions are directed inwards towards the magnetic axis, hence optimizing the absorbed power. In contrast, with counter-current injection, the first orbits of the fast ions are outwards, resulting in much greater prompt losses of the beam ions. These enhanced prompt losses result in a substantial reduction in the absorbed power with counter-injection compared with co-injection. The torque from the beams is, however, comparable to co- and counter-injection. This is because the $j_{r} \times B$ torque associated with the prompt losses is larger with counter-injection and compensates the reduction in the fast-ion absorption. Counterinjection also produces a broader torque deposition profile than co-injection because the fast-ion losses increase towards the edge.

The evolution of two discharges with co- and counter-NBI heating ( $\sim 3 \mathrm{MW}$ of $\mathrm{D}^{0}$ at $\left.60 / 68 \mathrm{keV}\right)$ is shown in figure 1 . The plasma current is ramped at $3.5 \mathrm{MA} \mathrm{s}^{-1}$ reaching $850 \mathrm{kA}$ at $0.2 \mathrm{~s}$ (the toroidal field $B_{\mathrm{t}} \sim 0.53 \mathrm{~T}$ ). The gas fuelling rate in both discharges is the same. As can be seen from the evolution of the line-average density $\bar{n}_{\mathrm{e}}$, the particle confinement in the counter-NBI discharge is greater, with $\bar{n}_{\mathrm{e}}$ about $30 \%$ higher than in the co-NBI discharge in the period with one beam. After this time when the second beam is injected, both the density 

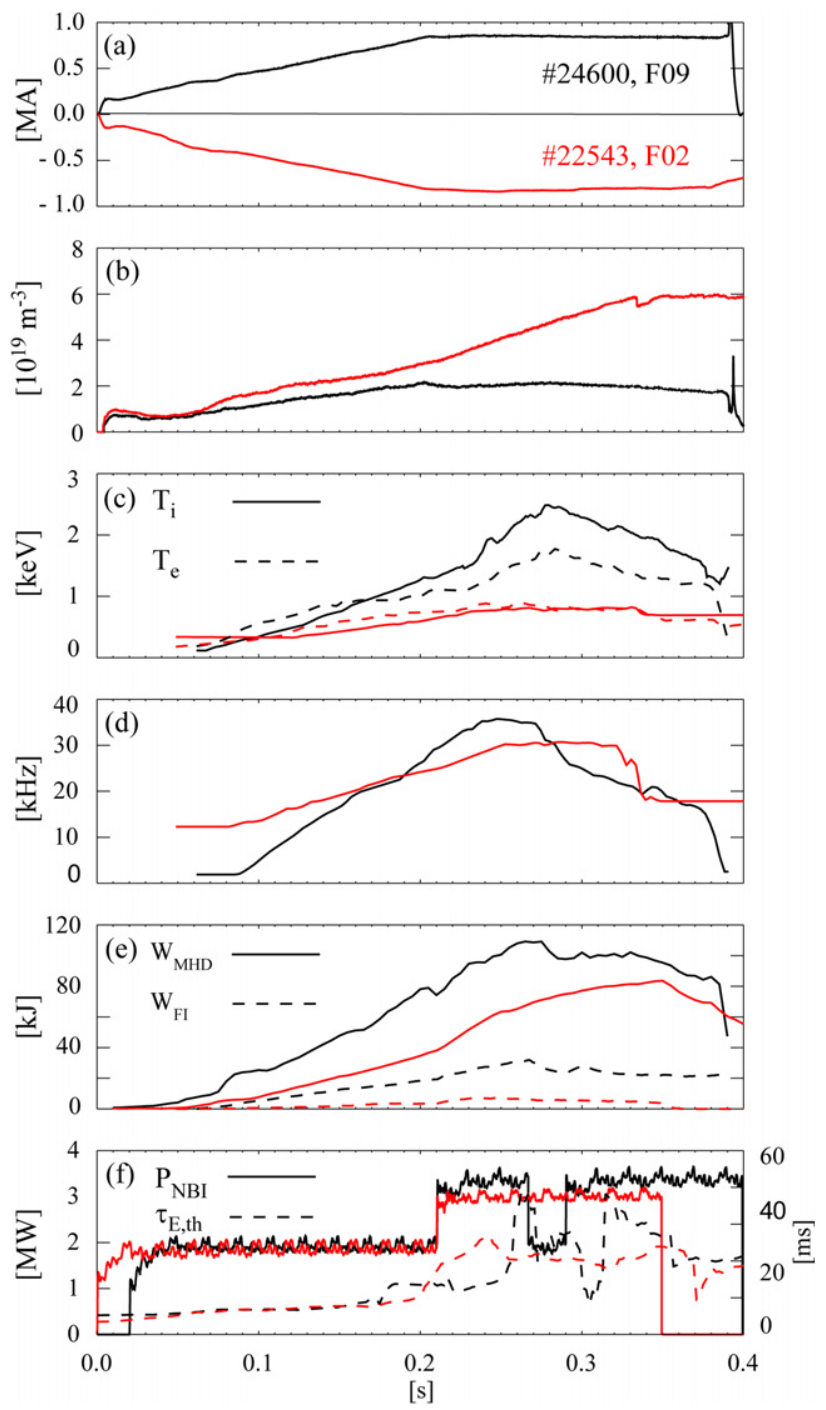

Figure 1. Evolution of discharges with co- (\#24600, black) and counter-current (\#22543, red) directed NBI heating showing: $(a)$ plasma current, $(b)$ line-averaged density, $(c)$ central ion (solid) and electron (dashed) temperatures, $(d)$ central toroidal rotation frequency, $(e)$ total (solid) stored energy and fast-ion energy (dashed) and $(e)$ injected NBI power (solid) and thermal energy confinement times (dashed).

and stored energy $W_{\mathrm{MHD}}$ increase strongly in the counterNBI discharge, the increase in $W_{\text {MHD }}$ mainly being due to an increase in $\bar{n}_{\mathrm{e}}$ which is a factor of $\sim 3$ times that in the co-current discharge by the end of the flat-top phase.

As can be seen from figure $1(f)$, in the period with $3 \mathrm{MW}$ of NBI the energy confinement time $\tau_{\mathrm{th}, E}$ in both discharges increases, with the largest increase by a factor of $\sim 2$ in the counter-NBI discharge being due to the density increase. In the co-NBI discharge the central temperatures increase in the high-power phase, with $T_{\mathrm{i}}(0)$ reaching $2.5 \mathrm{keV}$ and $T_{\mathrm{i}}>T_{\mathrm{e}}$, while in the counter-NBI discharge the temperatures do not exceed $1 \mathrm{keV}$ and $T_{\mathrm{i}} \sim T_{\mathrm{e}}$. The fast-ion energy content $W_{\mathrm{FI}}$ of the co-NBI discharge is about $30 \%$ of the total while in the counter-NBI discharge this is less than $10 \%$, which is consistent with the much reduced absorbed power fraction with counter-injection, which is $\sim 0.5$ compared with $\sim 0.8$ with co-injection. Although the density is higher in the counter-
NBI discharge, the toroidal rotation frequency $f_{\phi}=\omega_{\phi} / 2 \pi$ achieved of $\sim 30 \mathrm{kHz}$ is comparable to that with co-injection. This is because, although the absorbed power is less with counter-NBI, the applied torque is comparable.

The kinetic profiles measured during the two discharges are compared in figure 2 at representative times of $0.2 \mathrm{~s}$ at the end of the current ramp in the period with one beam, at 0.25 early in the period with two beams and later in the flattop phase at $0.3 \mathrm{~s}$. Only in the co-NBI discharge \#24600 was the new high-resolution NdYAG TS system [3] available for the $T_{\mathrm{i}}$ and $n_{\mathrm{e}}$ measurements. All the profiles have the peaked form typical of L-mode discharges. In the co-NBI discharge, the $T_{\mathrm{i}}$ and $\omega_{\phi}$ profiles exhibit a distinct increase in gradient or 'knee' at a normalized radius of $\rho \sim 0.4$, where the normalized gradients $\rho_{\mathrm{s}} / L_{T_{\mathrm{i}}}$ and $\rho_{\mathrm{s}} / L_{\omega}$ reach a maximum (where $\rho_{\mathrm{s}}$ is the ion Larmor radius at the sound speed and $\left.L_{T_{\mathrm{i}}}=\left(T_{\mathrm{i}}^{\prime} / T_{\mathrm{i}}\right)^{-1}\right)$, as shown in figure $2(c)$. This feature is particularly marked in the $\omega_{\phi}$ profile, which also exhibits a flattening at midradius. This strong increase in $\rho_{\mathrm{s}} / L_{T_{\mathrm{i}}}$ and $\rho_{\mathrm{s}} / L_{\omega}$ is indicative of the formation of an ITB in the ion thermal and momentum channels. There is no such feature evident in the $T_{\mathrm{e}}$ profiles indicating that no ITB forms in the electron channel.

With counter-NBI all the kinetic profiles are generally broader than with co-NBI. There is not such clear evidence of the presence of an ITB as with co-NBI; however, in the later period with $\sim 3 \mathrm{MW}$ of NBI when the density increases, a local maximum in $\rho_{\mathrm{S}} / L_{T_{\mathrm{i}}}$ does develop at $\rho \sim 0.4$ indicating the formation of barrier in the ion thermal channel. The density profile also peaks in this period exhibiting a maximum in $\rho_{\mathrm{s}} / L_{n_{\mathrm{e}}}$ at $\rho \sim 0.3$ The $\omega_{\phi}$ profile is broader than with co-NBI, with a more uniform gradient, although there are some weaker localized maxima evident in $\rho_{\mathrm{s}} / L_{\omega}$. The formation and evolution of the ITBs in these discharges is discussed in greater detail below.

\subsection{Co-NBI discharge}

3.2.1. ITB evolution. The evolution of the ion thermal and momentum ITBs in the discharge with co-NBI heating is best discussed with reference to that of the normalized gradients $\rho_{\mathrm{s}} / L_{T_{\mathrm{i}}}$ and $\rho_{\mathrm{s}} / L_{\omega}$, which are shown in figure 3 along with the evolution of the magnetic shear $\hat{s}$ and the location of $q_{\min }$ where $\hat{s}=0$. Because MHD activity has a strong influence on the rotation profile a magnetic spectrogram from an outboard Mirnov coil and the profile evolution of the rate of change in toroidal rotation $\dot{\omega}_{\phi}$ are also shown in figure 3. The ITB in the ion thermal channel forms at about $0.1 \mathrm{~s}$ when a local maximum in $\rho_{\mathrm{s}} / L_{T_{\mathrm{i}}}$ develops at $q_{\min }$ at about the time at which $q_{\min }$ reaches 3 . In this region the ion thermal diffusivity $\chi_{i}$ locally falls to the ion neo-classical value as discussed in more detail below. At this time the core $T_{\mathrm{i}}$ is only about $300 \mathrm{eV}$, although the normalized ion temperature gradient (ITG) $\rho_{\mathrm{S}} / L_{T_{\mathrm{i}}}$ (where $\rho_{\mathrm{s}}$ is the ion Larmor radius at the sound speed and $\left.L_{T_{\mathrm{i}}}=\left(T_{\mathrm{i}}^{\prime} / T_{\mathrm{i}}\right)^{-1}\right)$ already reaches 0.1 . An ITB in the momentum channel forms before this, at the onset of negative magnetic shear at $\sim 0.07 \mathrm{~s}$, with a peak in $\rho_{\mathrm{S}} / L_{\omega}$ localized to the region of most negative magnetic shear a few $\mathrm{cm}$ inside $q_{\mathrm{min}}$. This is stronger than the ion thermal ITB with the maximum of $\rho_{\mathrm{S}} / L_{\omega} \sim 0.2$ located inside the peak of $\rho_{\mathrm{s}} / L_{T_{\mathrm{i}}}$. There is no sign of ITB formation in the electron 

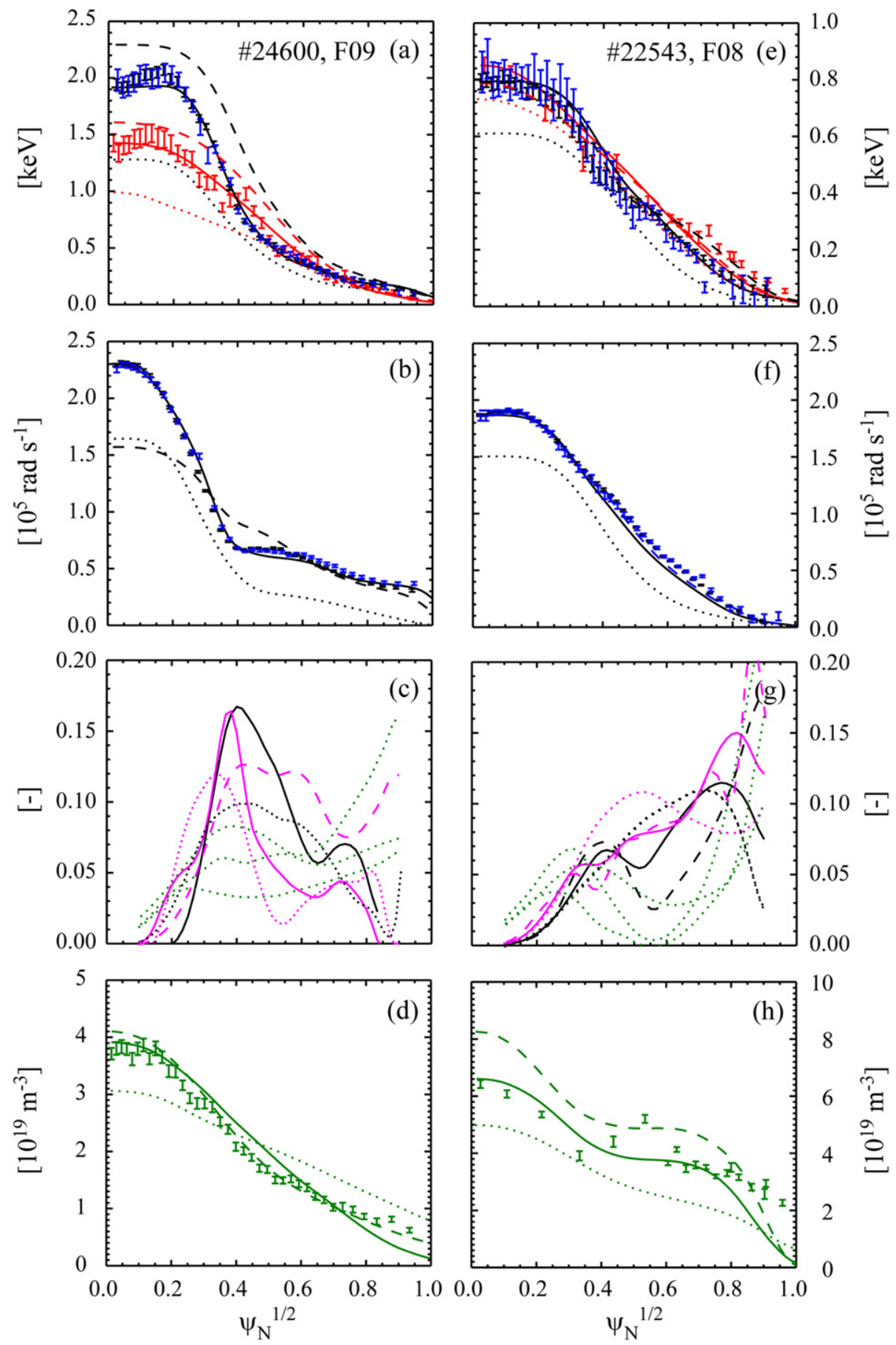

Figure 2. Kinetic data at $0.2 \mathrm{~s}$ (dotted), $0.25 \mathrm{~s}$ (solid) and $0.3 \mathrm{~s}$ (dashed) during two MAST discharges with co- $((a)-(d)$, \#24600) and counter-current $((e)-(h), \# 22543)$ oriented NBI heating: $(a),(e)$ temperatures $T_{\mathrm{i}}$ (black) from CXRS (black points viewing the SS beam and blue points viewing the SW beam) and $T_{\mathrm{e}}$ (red) from NdYAG TS; $(b),(f)$ toroidal rotation rate $\omega_{\phi}$ from CXRS; normalized gradients $\rho_{\mathrm{s}} / L_{T_{\mathrm{i}}}$ (black), $\rho_{\mathrm{s}} / L_{\omega_{\phi}}$ (magenta) and $\rho_{\mathrm{s}} / L_{n_{\mathrm{e}}}$ (green); and $(d),(h)$ electron density $n_{\mathrm{e}}$.

channel, as the $T_{\mathrm{e}}$ profile is broad with the gradient distributed over the full radial region outside $q_{\mathrm{min}}$. The formation of the ITB is better illustrated in figure 4 , which shows the evolution of the maxima of $\rho_{\mathrm{s}} / L_{T_{\mathrm{i}}}$ and $\rho_{\mathrm{s}} / L_{\omega_{\phi}}$ and their radial locations, which are close to that of $q_{\mathrm{min}}$. The times when $q_{\mathrm{min}}$ reaches rational values are also indicated. These times show some correlation with temporary increases in $\rho_{\mathrm{S}} / L_{\omega_{\phi}}$ and temporary reductions in $\rho_{\mathrm{S}} / L_{T_{\mathrm{i}}}$, which increases in the periods between the integer- $q$ crossings. Such behaviour has also been observed on DIII-D [17].

Once the ITB is established $T_{\mathrm{i}}$ and $\omega_{\phi}$ both increase rapidly inside $q_{\min }$, which is located at about $\rho \sim 0.5$, with $T_{\mathrm{i}}(0)$ increasing to $\sim 2.5 \mathrm{keV}$ and $\omega_{\phi}(0)$ to $\sim 2 \times 10^{5} \mathrm{~s}^{-1}$, while $T_{\mathrm{e}}(0)$ reaches $\sim 1.5 \mathrm{keV}$. This rotation rate corresponds to a toroidal Mach number $M_{\phi} \sim 0.5$. The stored energy $W_{\mathrm{MHD}}$ reaches $\sim 100 \mathrm{~kJ}$, including that of the fast ions, which is 


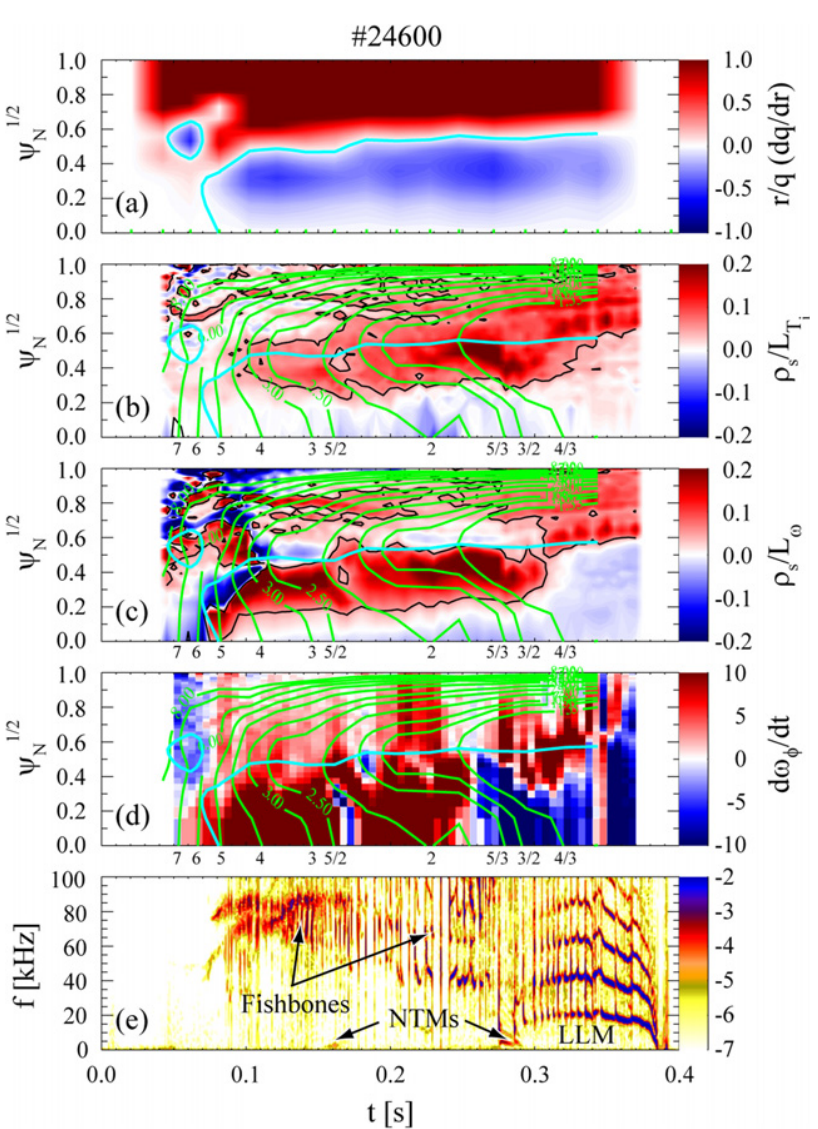

Figure 3. Evolution of $(a)$ magnetic shear $\hat{s},(b)$ normalized ITG $\rho_{\mathrm{s}} / L_{T_{\mathrm{i}}}$ and $(c)$ toroidal rotation $\rho_{\mathrm{s}} / L_{\omega}$ gradient, $(d)$ rate of change in toroidal rotation $\dot{\omega}_{\phi}$ and $(e)$ MHD in co-NBI ITB discharge \#24600. Locations of rational surfaces (green, labelled below plots) and $q_{\min }$ (cyan) are also shown.

comparable to the thermal energy content of these low-density plasmas.

3.2.2. Transport analysis. Thermal transport analysis requires knowledge of the heating profiles. The deposition of the dominant NBI heating is determined in TRANSP [6] using the NUBEAM Monte Carlo model, which assumes classical diffusion and slowing down of the fast ions. Several factors indicate that, under some conditions, the loss rate of fast ions must exceed that due to classical diffusion, primarily due to fast-ion driven MHD activity. Figure 5 shows a comparison between various measured quantities which are sensitive to the level of fast-ion losses and the results of transport analysis using TRANSP [6]. These include the total D-D neutron rate $R_{\mathrm{N}}$, the total stored energy $W_{\mathrm{MHD}}$ and fast-ion stored energy $W_{\mathrm{FI}}$, the Shafranov shift of the magnetic axis $\Delta R_{\mathrm{Sh}}$ and the absorbed NBI power $P_{\mathrm{NI}}^{\mathrm{abs}}$. Both $W_{\mathrm{MHD}}$ and $\Delta R_{\mathrm{Sh}}$ are available from EFIT equilibrium reconstructions. The standard EFIT is constrained only to magnetic data while a more accurate reconstruction can be obtained using additional constraints to the MSE magnetic pitch angle data and to the total pressure, including that of the fast ions, which is available from an initial TRANSP run. This can be performed using the $\mathrm{MC}^{3}$ analysis chain and requires iteration to include the fast-ion pressure self-consistently.
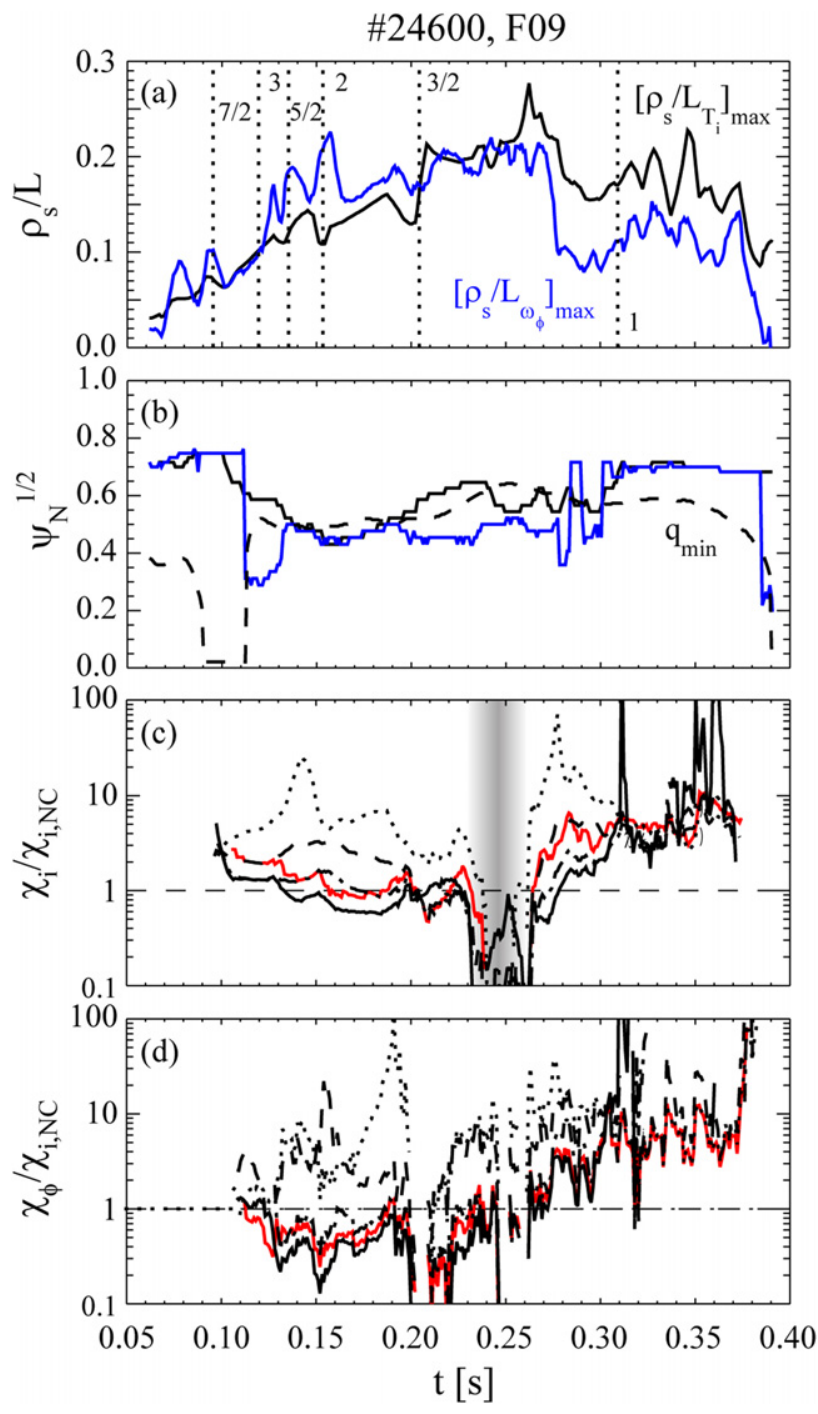

Figure 4. Evolution of the discharge shown in figure 1 showing: $(a)$ the maximum values of $\rho_{s} / L_{T_{\mathrm{i}}}$ (black), $\rho_{\mathrm{s}} / L_{\omega_{\phi}}$ (blue) and $(b)$ their radial locations along with that of $q_{\min }$ (dashed); (c) the ratio

$\chi_{\mathrm{i}} / \chi_{\mathrm{i}, \mathrm{NC}}$ at the location of $\left(\rho_{\mathrm{s}} / L_{T_{\mathrm{i}}}\right)_{\max }(\mathrm{red})$ and at fixed normalized radii (black) of $\rho=0.4$ (solid), 0.5 (dotted-dashed), 0.6 (dashed) and 0.7 (dotted); and $(d)$ the ratio $\chi_{\phi} / \chi_{\mathrm{i}, \mathrm{NC}}$ at the location of $\left(\rho_{\mathrm{s}} / L_{\omega_{\phi}}\right)_{\max }$ with the same key as in $(c)$. Times when $q_{\min }$ crosses rational values are also shown in $(a)$.

As can be seen from figure 5, in the co-NBI discharge \#24600 during the period with two beams the plasma energy $W_{\text {MHD }}^{\text {EFIT }}$ estimated from EFIT is lower than that calculated by TRANSP, the latter overestimating $W_{\mathrm{MHD}}$ by up to a factor of $\sim 1.3$ compared with that calculated by the standard EFIT. Overestimating the total pressure also results in a Shafranov shift which is up to $10 \mathrm{~cm}$ larger than the value obtained from the standard EFIT. A further consequence is that the predicted neutron rate is up to a factor of $\sim 2$ too high compared with that measured using a fission chamber. These factors together indicate that an appreciable anomalous loss of fast ions must be occurring during the period after $0.21 \mathrm{~s}$ with $\sim 3 \mathrm{MW}$ of NBI heating. In the period prior to this there is much better agreement between the measurements and TRANSP simulation without having to invoke these losses.

There is a simple ad hoc model of anomalous fast-ion diffusion in TRANSP, which assumes a diffusion coefficient 

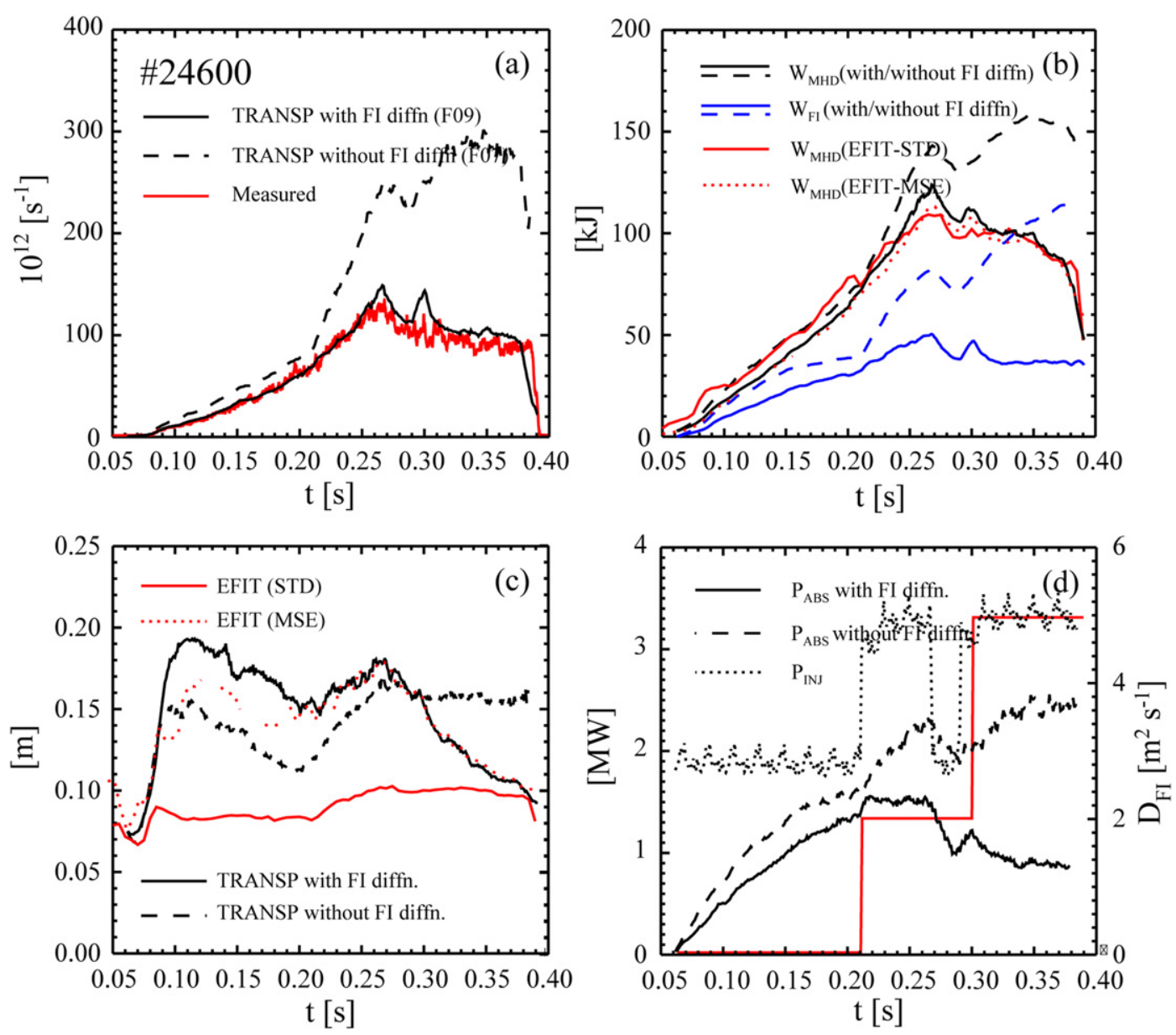

Figure 5. Comparison of the evolution of several parameters sensitive to the level of anomalous fast-ion losses for discharge \#24600 with co-NBI heating: (a) total neutron rate (red-measured and black-from TRANSP simulations, both with (solid) and without (dashed) anomalous fast-ion losses); $(b)$ total stored energy $W_{\text {MHD }}$ from EFIT (red) and from the TRANSP simulation (black) and the fast-ion stored energy (blue); $(c)$ Shafranov shift of magnetic axis; and $(d)$ injected (dotted) and absorbed (solid/dashed) NBI power and assumed fast-ion diffusion coefficient, $D_{\mathrm{FI}}$ (red). Results from both the 'standard' magnetics-only EFIT reconstruction (solid) and constrained by the MSE data (dotted) are shown.

$D_{\text {fast }}$ which is isotropic in pitch angle, with prescribed radial and energy dependences. It is necessary to assume additional fast-ion losses in discharge \#24600 only after $0.21 \mathrm{~s}$ when the NBI power is increased from $\sim 1.8 \mathrm{MW}$ to $\sim 3.2 \mathrm{MW}$, indicating that there is a strong power dependence of the losses. In this phase a value of $D_{\text {fast }} \sim 3 \mathrm{~m}^{2} \mathrm{~s}^{-1}$ is required and later, following the onset of an $n=1$ internal kink mode in the core after $0.3 \mathrm{~s}$, an even higher value $\sim 5 \mathrm{~m}^{2} \mathrm{~s}^{-1}$ has to be assumed. Here, anomalous diffusion is applied only to fast ions with energies above $30 \mathrm{keV}$, which reduces the neutron rate $R_{\mathrm{N}}$ by a larger factor than the stored energy $W_{\text {MHD }}$ because the D-D fusion cross-section is larger at higher energies. The resulting increase of fast-ion losses results in a substantial reduction in the absorbed NBI power by factors of $\sim 0.4(0.21$ to $0.3 \mathrm{~s})$ and $\sim 0.5$ (after $0.3 \mathrm{~s}$ ) in these two phases, respectively.

With these assumptions on the anomalous fast-ion diffusion there is much better agreement between predicted values of $W_{\mathrm{MHD}}$ and $\Delta R_{\mathrm{Sh}}$ and those obtained from EFIT reconstructions constrained to the MSE data and total pressure, as well as between the measured and predicted neutron rate. In the early phase, however, before $0.21 \mathrm{~s}$ when anomalous losses do not have to be assumed, TRANSP over predicts $\Delta R_{\mathrm{Sh}}$ by a few $\mathrm{cm}$ indicating that the total pressure may be less peaked than in the TRANSP simulation and that some level of fast-ion redistribution may be occurring in the core plasma. There is insufficient data available, however, to constrain a more detailed model of fast-ion diffusion. To address this issue, the MAST team has recently implemented new diagnostics to diagnose the behaviour of the fast ions in more detail, including a multi-channel neutron camera and a fast-ion $\mathrm{D}_{\alpha}$ spectroscopy (FIDA) system.

The evolution of the ion thermal diffusivity normalized to the neo-classical value $\chi_{\mathrm{i}} / \chi_{\mathrm{i}, \mathrm{NC}}$ is shown in figure $4(c)$ at the location of the ITB, i.e. the maximum of $\rho_{\mathrm{S}} / L_{T_{\mathrm{i}}}$, and at four representative normalized radii, $\rho=0.4,0.5,0.6$ and 0.7 . In the initial phase heated with one beam, when it is not necessary to invoke the anomalous fast-ion losses, the inferred level of ion thermal transport at the ITB is close to the neo-classical value with $\chi_{\mathrm{i}} / \chi_{\mathrm{i}, \mathrm{NC}} \sim 1$. The ITB is located in the region of negative magnetic shear inside $\rho \sim 0.5$. Outside $q_{\min }$, in the positive shear region, $\chi_{i}$ considerably exceeds the neo-classical value, e.g. by an order of magnitude at $\rho \sim 0.7$. In the latter phase $(>0.21 \mathrm{~s})$ with two beams when the anomalous fast-ion losses have to be invoked, there is a period $(0.23-0.27 \mathrm{~s})$ exhibiting negative values of the ion heat flux $q_{\mathrm{i}}$ and hence $\chi_{\mathrm{i}}$ (indicated by the shaded region). This occurs because the magnitude of $q_{\mathrm{i}}$ 
is relatively small compared with the total heat flux and hence sensitive to systematic uncertainties in the power deposition profile, which is in turn dependent on the rather crude ad hoc fast-ion redistribution model, and also to rapid changes in the kinetic energy $W_{\text {kin }}$ at this time.

Momentum transport is also analysed in TRANSP which calculates the time-dependent balance of toroidal angular momentum: $i_{\phi}=-\nabla \cdot \Gamma_{\phi}+T_{\phi}$, where the angular momentum density is given by $l_{\phi}=m_{\mathrm{i}} n_{\mathrm{i}}\left\langle R^{2}\right\rangle \omega_{\phi}$ and the momentum flux can be represented as $\Gamma_{\phi}=-m_{\mathrm{i}} n_{\mathrm{i}}\left\langle R^{2}\right\rangle \chi_{\phi}^{\text {eff }} \mathrm{d} \omega_{\phi} / \mathrm{d} r$, where $\chi_{\phi}^{\text {eff }}$ is the effective momentum diffusivity. In this form, $\chi_{\phi}^{\text {eff }}$ represents the net momentum transport as a diffusivity, including implicitly any contribution from a pinch term which would be proportional to $\omega_{\phi}$ rather than $\mathrm{d} \omega_{\phi} / \mathrm{d} r$. It is not possible, however, to determine the diffusive and pinch contributions separately without performing dedicated experiments. The net torque $T_{\phi}$ includes both the collisional and prompt torque from the NBI heating and the contributions from friction with neutral atoms and charges exchange losses.

Figure $4(d)$ shows the evolution of $\chi_{\phi}^{\text {eff }} / \chi_{i}^{\mathrm{NC}}$ from this analysis at the ITB, i.e. the maximum of $\rho_{\mathrm{s}} / L_{\omega_{\phi}}$, and at four representative normalized radii, $\rho=0.4,0.5,0.6$ and 0.7 . As for the thermal transport analysis, the need to assume anomalous fast-ion losses in the period with two beams after $0.21 \mathrm{~s}$ makes the sources uncertain. Prior to this when these losses do not have to be assumed, at the location of the ITB, $\chi_{\phi}^{\text {eff }}$ is somewhat below the level of the neo-classical thermal diffusivity, consistent with there being an ITB in the momentum channel there, whereas further outside at $\rho \sim 0.7, \chi_{\phi}^{\text {eff }}$ considerably exceeds $\chi_{i}^{\mathrm{NC}}$. The observation that $\chi_{\phi}^{\text {eff }} / \chi_{\mathrm{i}}^{\mathrm{NC}}<1$ in the ITB region is not unphysical because, as discussed in more detail in section 3.3, the neo-classical prediction for the momentum diffusivity is smaller than that for the thermal diffusivity by at least a factor $\varepsilon^{3 / 2}$ (which is $\sim 0.1$ at the ITB location) because trapped particles do not transport net toroidal momentum.

After the ITB has terminated following the onset of an internal kink mode, which is discussed in more detail below, the rotation profile broadens and the core plasma inside the $q=1$ surface rotates as a rigid body. In this latter phase both $\chi_{\mathrm{i}}$ and $\chi_{\phi}^{\text {eff }}$ exceed the ion neo-classical value by up to an order of magnitude over the full plasma radius. This implies that the reduced level of flow shear caused by the presence of the kink mode results in a higher level of anomalous turbulent transport.

3.2.3. MHD behaviour. During the period from 0.07 to $0.16 \mathrm{~s}$, during which the ITB forms, there is strong chirping TAE mode activity driven by the fast-ion pressure gradient. This activity is not classical 'fishbone' modes, which require $q_{\text {min }}$ to be close to unity, but fast-ion driven, internal kink modes associated with higher order rational surfaces. At this time the rotation exhibits an inverted (positive) gradient just outside $q_{\text {min }}$ (see figure 3(c)). Although this gradient reversal reduces $\rho_{\mathrm{s}} / L_{\omega}$ at $q_{\mathrm{min}}$, it appears to enhance the rotation gradient just inside $q_{\min }$ in the region of favourable negative magnetic shear, hence locally increasing the $E \times B$ shearing rate $\gamma_{E}$, which is proportional to $L_{\omega}^{-1}$. This may therefore facilitate formation of the ITB and perhaps also help localize it to the region inside $q_{\text {min }}$.
It is interesting to consider what conditions could produce this reversal of the rotation gradient. Integrating the momentum balance equation, for simplicity in cylindrical coordinates but including a pinch term, results in the following expression:

$$
\begin{aligned}
\frac{\mathrm{d} \omega_{\phi}}{\mathrm{d} r} & =\frac{1}{\chi_{\phi}}\left[\frac{1}{I_{\phi} r} \frac{\mathrm{d}}{\mathrm{d} t} \int_{0}^{r} I_{\phi} r \omega_{\phi} \mathrm{d} r+\omega_{\phi} V_{\text {pinch }}\right. \\
& \left.-\frac{1}{I_{\phi} r} \int_{0}^{r} r T_{\phi} \mathrm{d} r\right]
\end{aligned}
$$

where $I_{\phi}=m_{\mathrm{i}} n_{\mathrm{i}}\left\langle R^{2}\right\rangle$ is the moment of inertia 'density' and $V_{\text {pinch }}$ is the momentum pinch velocity. From this, three conditions required to produce a locally positive rotation gradient at radius $r$ can be discerned (the first two in the absence of a pinch): (i) if the rate of change in angular momentum inside the flux surface were to exceed the applied torque (this could only be transitory and it is difficult to perceive of how this could occur), (ii) in steady-state, if the net torque inside the flux surface were to become negative and (iii) if there were a strong outward momentum pinch at the location of gradient reversal. In non-circular geometry, shape changes in the flux surfaces might also play a role, although also in a transitory manner.

A localized counter-current (negative) torque could arise from an outward redistribution of the fast ions by the TAE MHD activity, which would be balanced by an inward radial return current resulting in a $j_{r} \times B_{\theta}$ torque counter to the plasma current. For this to result in the observed localized gradient reversal, the total $j_{r} \times B_{\theta}$ torque would have to exceed the applied NBI torque inside $q_{\text {min }}$. Another possible mechanism for producing a negative torque inside $q_{\text {min }}$ might be neoclassical toroidal viscosity (NTV) breaking [21] resulting from the plasma flowing through a non-axisymmetric kink perturbation of the plasma produced by the TAE modes. Detailed analysis and modeling is required to further elucidate the cause of this gradient reversal and to determine whether the observed MHD activity could produce a breaking torque of the required magnitude.

The strong pressure gradient at the ITB causes growth of NTM instabilities by increasing the drive from the bootstrap current. A modest decrease in the critical 'seed' island width is also expected due to the reduction in the perpendicular transport in the ITB region. This is because the seed island width is predicted to scale as $W_{\mathrm{d}} \propto\left(\chi_{\perp} / \chi_{\|}\right)^{1 / 4}$ [22]. Such a mode is observed in the discharge shown in figure 3 at about $0.16 \mathrm{~s}$, which causes a localized braking of rotation inside $q_{\mathrm{min}}$ and an acceleration further outside, due to coupling with another mode of different mode numbers there. This weakens the gradients at the ITB causing the mode to die away, allowing the ITB to be sustained and to increase in strength later on. Another tearing mode consequently occurs later in the discharge at $0.28 \mathrm{~s}$, which causes a similar localized braking of the core rotation.

An example of such coupling of $n=3$ and $n=2$ tearing modes at $q=4 / 3$ and $3 / 2$ surfaces, respectively, is shown in figure 6 , where the three independent measurements of the MHD frequency, rotation rate and the $q$-profile are all consistent. (The toroidal mode numbers $n$ determined from the Mirnov coil arrays are also consistent.) The condition where the modes at frequencies $\omega_{1,2}=\omega\left(\rho_{1,2}\right)+\omega_{\mathrm{m}, 1,2}$ 


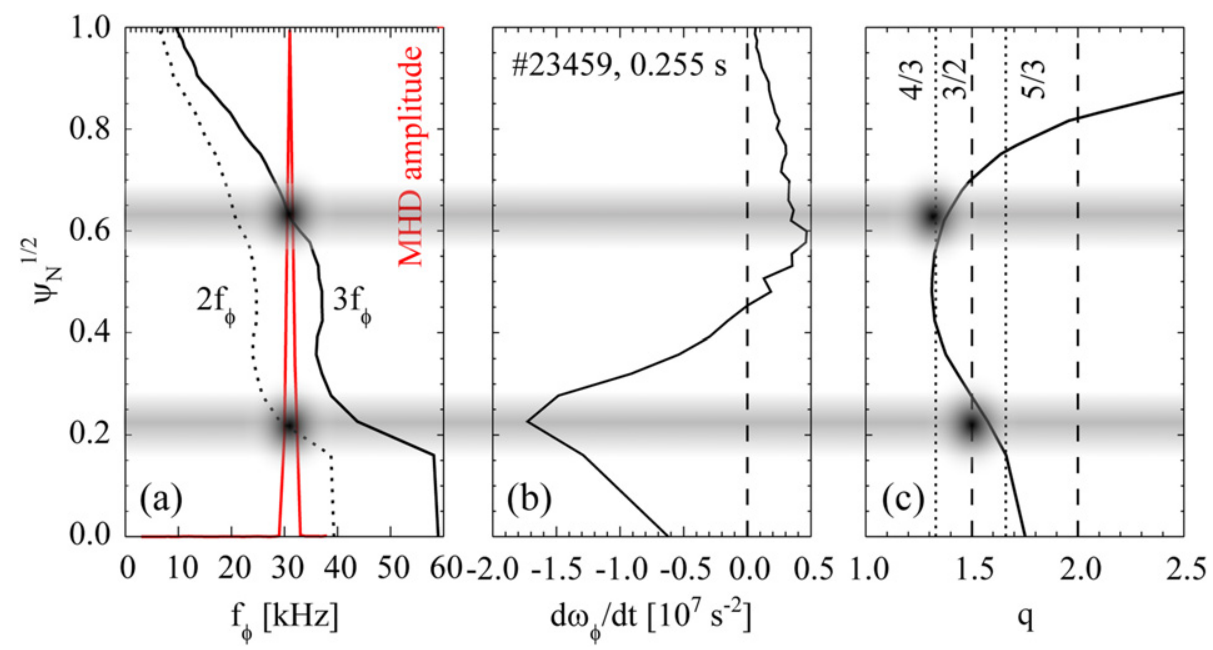

Figure 6. Example of coupling of $n=3$ mode at $q=4 / 3$ surface to $n=2$ mode at $q=3 / 2$ showing: $(a) n \times \omega_{\phi}$ and MHD spectrum, $(b)$ torque $\propto \mathrm{d} \omega_{\phi} / \mathrm{d} t,(c) q$-profile.

(where $\omega(\rho)$ is the rotation rate where the mode is resonant and $\omega_{\mathrm{m}}$ is the mode propagation frequency) are matched is $n_{1}\left(\omega\left(\rho_{1}\right)+\omega_{\mathrm{m}, 1}\right)=n_{2}\left(\omega\left(\rho_{2}\right)+\omega_{\mathrm{m}, 2}\right)$. When $\omega_{\mathrm{m}} \ll \omega(\rho)$, the coupling condition is approximately $n_{1} \omega\left(\rho_{1}\right)=n_{2} \omega\left(\rho_{2}\right)$. In figure 6 modes resonant at $q\left(\rho_{1,2}\right)=n_{1,2} / m_{1,2}=4 / 3$ and $3 / 2$ apparently lock together in this way such that their observed frequencies are the same. Localized breaking at the $q=3 / 2$ surface and acceleration at the $q=4 / 3$ surface is observable from the rate of change in toroidal rotation as shown in figure $6(b)$.

A tentative explanation of how such a mode coupling could occur is that the field perturbation due to one mode $\delta B_{r 1}\left(\rho_{2}\right)$ and the perturbed current density inside the island of the other $\delta j_{\| 2}\left(\rho_{2}\right)$ could produce a net $\left\langle\delta j_{\|} \times \delta B_{r}\right\rangle$ force integrated over the flux surface. Although this force would be finite only if the helicity of the two modes was the same, i.e. $m_{1}=m_{2}$ and $n_{1}=n_{2}$, coupling could be mediated through $m \pm 1$, $n \pm 1$ side bands generated by the shaping of the flux surfaces and interaction with a $1: 1$ mode, which is simultaneously present.

This mode coupling does not lead to termination of the ITB, which occurs somewhat later. Once the central $q$ value $\left(q_{0}\right)$ approaches unity at about $0.31 \mathrm{~s}$, the plasma core becomes unstable to an internal $n=1, m=1$ kink mode, localized to the region where $q$ is less than a threshold value of $\sim 1.3$, which subsequently grows in amplitude and saturates [23]. This mode can be identified as the continuous mode at $\sim 20 \mathrm{kHz}$ present from $0.31 \mathrm{~s}$ until the termination of the discharge, which exhibits multiple toroidal harmonics. The resulting axially asymmetric perturbation causes braking of the core rotation, the magnitude of which has been shown to be consistent with the torque produced by NTV in the axially asymmetric field perturbation of the mode [21]. This braking flattens the rotation profile inside the $q=1$ surface and leads to termination of the ITB within about $30 \mathrm{~ms}$. Such modes, which are referred to on MAST as long-lived modes (LLMs) become unstable once $q_{0}$ falls below about 1.3. This is a somewhat higher threshold $q$ value than would be the case at larger aspect ratio [23].

\subsection{Counter-NBI discharge}

3.3.1. Discharge evolution. Heating by counter-current directed NBI is less efficient due to higher level of first-orbit losses of the fast ions. The beam torque, however, is of similar magnitude for a given injected power $P_{\text {inj }}$ because the countercurrent directed $j_{r} \times B$ torque due to the prompt fast-ion losses is in the same direction as the beam with counter-current NBI. Typically, the fraction of $P_{\text {inj }}$ absorbed by the plasma in these counter-NBI discharges is $\sim 0.5$ compared with over $\sim 0.8$ with co-injection, while the beam torque $\sim 1 \mathrm{Nm}$ is comparable. As shown in figure 1, the rotation rate achieved is therefore similar to that with co-NBI. Perhaps surprisingly, the peak stored energy $W_{\mathrm{MHD}} \sim 80 \mathrm{~kJ}$ is not greatly reduced compared with the similar co-NBI, hence the energy confinement with counter-NBI is improved relative to that with co-NBI.

As a result of these differences, the transport properties of the counter-NBI discharges are very different from those with co-NBI heating. Firstly, the temperatures in the counterdischarges are lower with $T_{\mathrm{i}} \sim T_{\mathrm{e}} \leqslant 800 \mathrm{eV}$ with broader profiles. Although the fuelling rates are comparable in the coand counter-NBI discharges, increased particle confinement with counter-NBI leads to up to a factor of $\sim 3$ higher lineaverage density in the later phase of the discharges. As in the co-NBI discharge, the magnetic shear is weakly negative in the core inside $\rho \sim 0.4$, as shown in figure 7(a). The lower temperature and higher density increases the ion-electron exchange power, this explaining the closeness of $T_{\mathrm{i}}$ and $T_{\mathrm{e}}$.

The counter-NBI discharges hence do not exhibit a distinct, localized ITB where $T_{\mathrm{i}}<T_{\mathrm{e}}$. There are however detailed, localized features on the $T_{\mathrm{i}}$ profile manifested as localized maxima in $\rho_{\mathrm{s}} / L_{T_{\mathrm{i}}}$, which can be related to the $q$-profile evolution. The rotation profile is also broad, with no clearly localized maxima in $\rho_{\mathrm{s}} / L_{\omega_{\phi}}$ but rather the gradient distributed over the whole profile. Transport analysis discussed below reveals that the momentum transport is below the level of neo-classical ion thermal transport over a wide region of the plasma and the ion thermal transport is also close to neo-classical in the plasma core. It is hence perhaps misleading to refer to these discharges as exhibiting ITBs in the usual sense. 


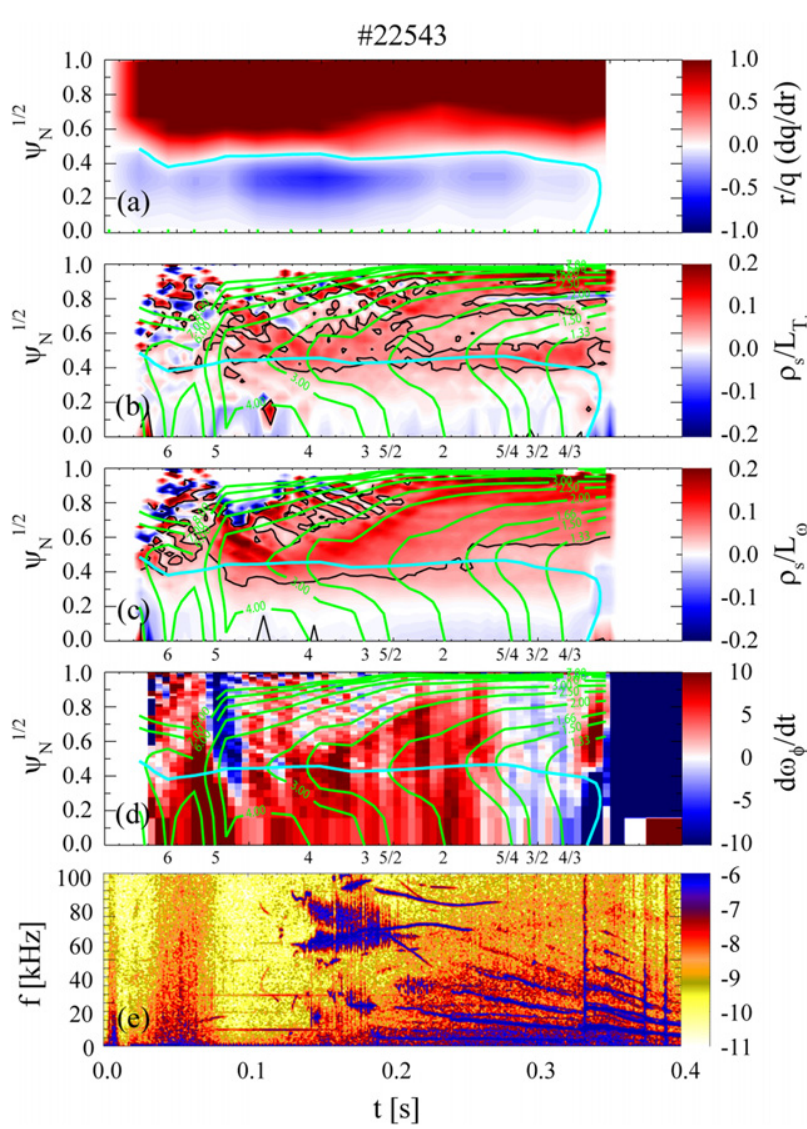

Figure 7. Evolution of a counter-NBI ITB discharge \#22543 with plots as defined in figure 1 .

Early in the discharge, the absorbed NBI power is very low, so it is difficult to determine the ion power and momentum balances with any degree of certainty in the TRANSP analysis. The CXRS rotation data show, however, that already at this early time, the counter-NBI discharge is rotating much faster than the co-NBI discharge. After about $0.1 \mathrm{~s}$, when appreciable NBI power is absorbed, as can be seen in figure 7(c), the normalized rotation gradient $\rho_{\mathrm{s}} / L_{\omega_{\phi}}$ increases just outside $q_{\text {min }}$ at about $\rho \sim 0.5$ in the region of positive magnetic shear. This follows a marked acceleration of the toroidal rotation of the core plasma inside $q_{\min }$ and a deceleration outside, as can be seen in figure $7(d)$. By the end of the current ramp at $0.2 \mathrm{~s}$, the rotation gradient has broadened across the whole outer region of the plasma, which is consistent with the broad profile of $j_{r} \times B$ torque from the beams. The normalized temperature gradient $\rho_{\mathrm{s}} / L_{T_{\mathrm{i}}}$ also increases just outside $q_{\min }$ at the same time as $\rho_{\mathrm{s}} / L_{\omega_{\phi}}$. As the rotation profile broadens, the localized maximum in $\rho_{\mathrm{s}} / L_{T_{\mathrm{i}}}$ at mid-radius appears to bifurcate, i.e. exhibit a second maxima, the outer maxima following the approximate location of the $q=5 / 2$ surface, while the inner remains at the location of $q_{\mathrm{min}}$.

From the onset of the period with two beams after $0.21 \mathrm{~s}$, the rate of rise in density and stored energy increases, with an associated increase in $\tau_{E \text {,th }}$, as shown in figure $1(f)$. This is a result primarily of a peaking of the $n_{\mathrm{e}}$ profile in the plasma core inside $\rho \sim 0.4$ and the formation of a significant density pedestal at the plasma periphery, although there is also some increase in $T_{\mathrm{i}}$ gradient in the core. There is no sign of ELM-like instabilities during this latter period. After $\sim 0.26$ s there is noticeable $n=1, m=1$ mode activity in the MHD spectrogram of similar character to the LLM observed in the co-NBI discharges. Perhaps associated with this MHD activity, a significant breaking of the rotation in the core plasma is observed at this time, as can be seen in figures $1(d)$ and $7(d)$.

3.3.2. Transport analysis. As with the co-NBI discharge, only in the latter period with $\sim 3 \mathrm{MW}$ of injected NBI power, is it is necessary to invoke some level of anomalous fastion diffusion to reconcile the predicted values of $R_{\mathrm{N}}, W_{\mathrm{MHD}}$ and $\Delta R_{\mathrm{Sh}}$ from the TRANSP calculations to the experimental values, although the initial discrepancy is not so great as within the co-NBI discharges. This is probably because the initial fast-ion pressure is much lower because of the high prompt fastion losses with counter-NBI heating, hence resulting in lower net losses for a given level of diffusion. Radially a constant value of $D_{\text {fast }}$ is assumed of $2 \mathrm{~m}^{2} \mathrm{~s}^{-1}$ after $0.21 \mathrm{~s}$, increasing to $4 \mathrm{~m}^{2} \mathrm{~s}^{-1}$ after $0.26 \mathrm{~s}$, again acting only on fast ions of energies above $30 \mathrm{keV}$.

As shown in figure 8 , the transport analysis shows that initially the momentum diffusivity $\chi_{\phi}^{\text {eff }}$ is at or below the ion neo-classical thermal diffusivity $\chi_{i}^{\mathrm{NC}}$ in the core region inside $\rho \sim 0.5$, then later on in the discharge, the region of reduced momentum transport broadens over most of the plasma radius. In the plasma core, the lowest values of $\chi_{\phi}^{\text {eff }}$ are an order of magnitude below $\chi_{i}^{\mathrm{NC}}$. This observation is not inconsistent with neo-classical theory, which predicts that in an up-down symmetric plasma there is no Pfirsh-Schlüter enhancement $\left(\sim q^{2}\right)$ of momentum transport as there is for thermal transport [24]. Also, because trapped particles do not carry toroidal momentum, there is no banana enhancement $\sim \varepsilon^{3 / 2}$ to the momentum diffusivity either. Hence, the neoclassical momentum diffusivity is smaller than the thermal diffusivity by up to a factor $\chi_{\phi}^{\mathrm{NC}} / \chi_{\mathrm{i}}^{\mathrm{NC}} \sim q^{2} / \varepsilon^{3 / 2}$, the term in $q$ depending on the degree of up-down asymmetry. Hence, $\chi_{\phi}^{\mathrm{NC}}$ can be at least an order of magnitude less than $\chi_{i}^{\mathrm{NC}}$ in the core region of these plasmas, where the observed $\chi_{\phi}^{\text {eff }}$ approaches this level.

In the initial phase with one beam before $0.21 \mathrm{~s}$, inside $\rho \sim 0.5$ in the region of negative magnetic shear, $\chi_{\mathrm{i}}$ is within a factor of $\sim 2$ of $\chi_{i}^{\mathrm{NC}}$ while outside of this it is up to an order of magnitude larger than $\chi_{i}^{\mathrm{NC}}$ increasing with radius. The maximum of $\rho_{\mathrm{s}} / L_{T_{\mathrm{i}}}$ is not within this region of suppressed ion thermal transport but is located further outwards at $\rho \sim 0.6$. During the later period with two beams from $0.21 \mathrm{~s}$, the ion thermal transport decreases further in the core and the region where $\chi_{i}<\chi_{i}^{N C}$ broadens further. After $0.27 \mathrm{~s}$, during the period of strong 1:1 MHD activity when the core rotation is braked, $\chi_{i}$ decreases well below the neo-classical level. This is likely to be non-physical and could be the consequence of viscous ion heating resulting from the rotation braking, which would not be included in the ion power balance in TRANSP.

\subsection{Magnetic shear dependence}

By varying the start time of the NBI heating during the initial current ramp it is possible to alter the degree of shear reversal in the core of co- and counter-NBI heated ITB discharges. Those with the earliest heating exhibit the most negative shear, while 

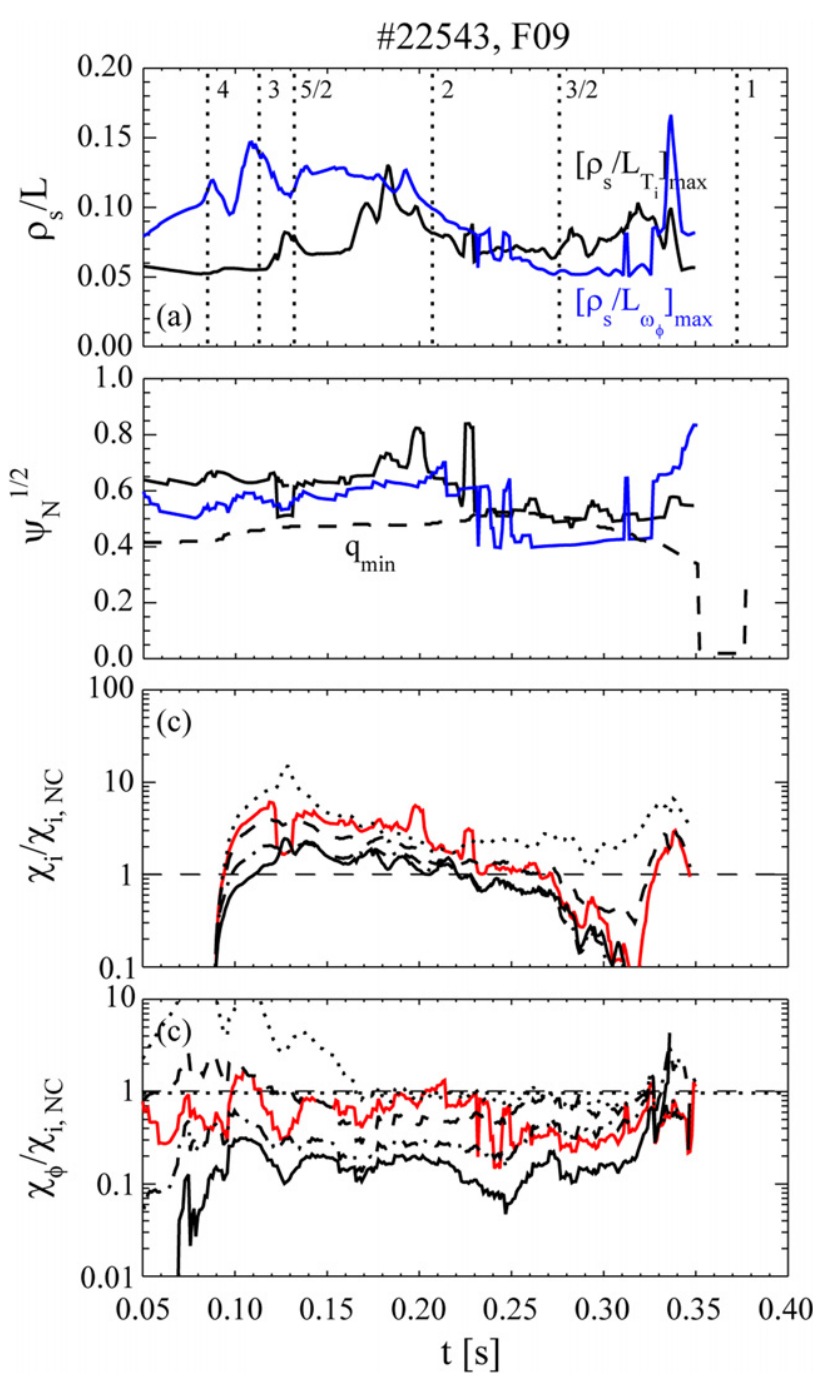

Figure 8. Evolution of discharge shown in figure 7 showing: $(a)$ the maximum values of $\rho_{s} / L_{T_{i}}$ (black), $\rho_{\mathrm{s}} / L_{\omega_{\phi}}$ (blue) and $(b)$ their radial locations along with that of $q_{\min }$ (dashed); $(c)$ the ratio $\chi_{i} / \chi_{\mathrm{i}, \mathrm{NC}}$ at the location of $\left(\rho_{\mathrm{s}} / L_{T_{\mathrm{i}}}\right)_{\max }(\mathrm{red})$ and at fixed normalized radii (black) of $\rho=0.4$ (solid), 0.5 (dot-dashed), 0.6 (dashed) and 0.7 (dotted); and $(d)$ the ratio $\chi_{\phi} / \chi_{\mathrm{i}, \mathrm{NC}}$ at the location of $\left(\rho_{\mathrm{s}} / L_{\omega_{\phi}}\right)_{\max }$ with the same key as in $(c)$. Times when $q_{\min }$ crosses rational values are also shown in $(a)$.

those with the NBI starting towards the end of the current ramp, exhibit only very weak negative or zero shear in the core. Data from several ITB discharges, five with co- and three with counter-NBI with different start times in the range $0.02-0.14 \mathrm{~s}$ (with the current ramp ending at $0.21 \mathrm{~s}$ ) are shown in figures $9(a)$ and $(b)$, which show the normalized gradients $\rho_{\mathrm{s}} / L_{T_{\mathrm{i}}}$ and $\rho_{\mathrm{s}} / L_{\omega_{\phi}}$ as a function of the magnetic shear. Data for each discharge are plotted for the range of normalized radii from $0.2<\rho_{\mathrm{n}}<0.7$, excluding the very core and outer regions, for times after formation of the ITBs and prior to the onset of the internal $n=1$ kink mode, which strongly modifies the rotation profile. With co-NBI heating, it can clearly be seen that the highest normalized rotation gradients $\left(\rho_{\mathrm{s}} / L_{\omega_{\phi}}>0.07\right)$ are localized to the negative shear region, indicating that the momentum transport is strongly reduced when $\hat{s}<0$. (There are also low values of the normalized gradients in the negative
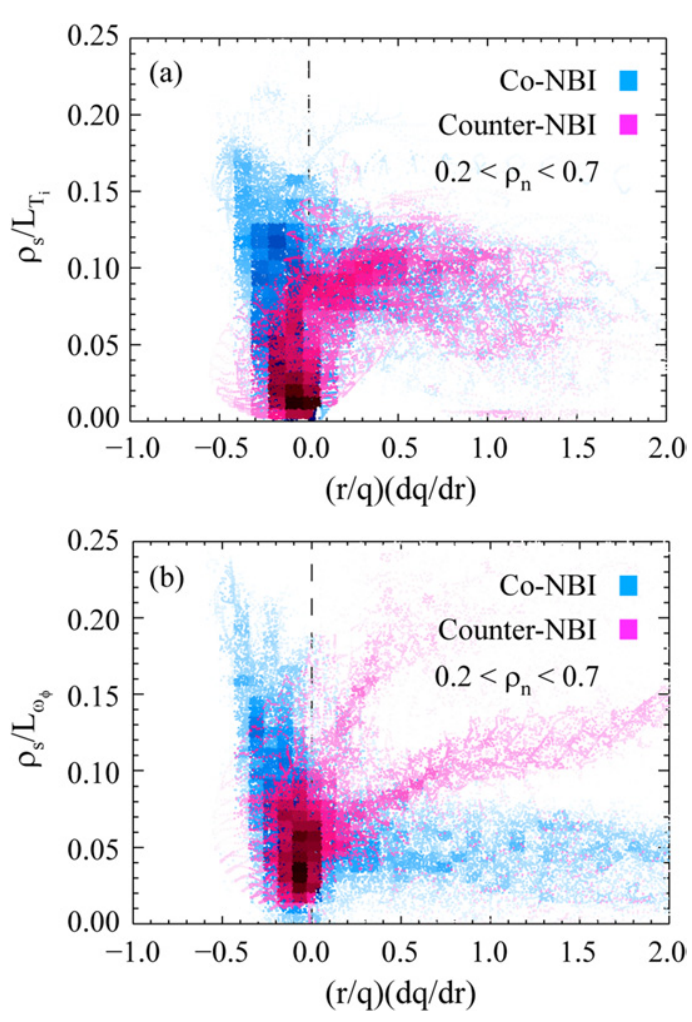

Figure 9. Dependence of normalized gradients of ion temperature $\rho_{\mathrm{s}} / L_{T_{\mathrm{i}}}(a)$ and rotation rate $\rho_{\mathrm{s}} / L_{\omega_{\phi}}(b)$ on magnetic shear $\hat{s}$ for several ITB discharges with co- or counter-NBI heating.

shear region because this includes data from inside the ITB in the plasma core where the gradients are weak.) The higher values of $\rho_{\mathrm{s}} / L_{T_{\mathrm{i}}}$ are somewhat less strongly localized to the region of negative magnetic shear than is the case for $\rho_{\mathrm{s}} / L_{\omega_{\phi}}$. This difference might be attributed to the fact that the neoclassical momentum diffusivity is smaller than the ion thermal diffusivity by the factor of at least $\chi_{\phi} / \chi_{\mathrm{i}} \sim \varepsilon^{3 / 2}$ (where $\varepsilon$ is the inverse aspect ratio $a / R$ ) because trapped particles cannot transport net toroidal momentum [24]. In the near absence of anomalous transport, the neo-classical transport is hence able to limit the maximum $T_{\mathrm{i}}$ gradient more effectively than that of the rotation in the ITB region.

With counter-NBI injection the region where the ion thermal transport is reduced to the neo-classical level (ITB) does not tend to form in the negative shear region, as in the case with co-NBI injection. Instead, the shear dependence shown in figure $9(b)$ is consistent with formation of the ITB in the vicinity of $q_{\min }(\hat{s} \sim 0)$ and its later evolution into the positive shear region. The bifurcation of the ITB discussed in section 2.3 is manifest in this figure as the second cluster of data at higher values of $\rho_{\mathrm{s}} / L_{\omega_{\phi}}$ in the positive shear region. The normalized rotation shear in the outer region is about twice that with co-NBI. This is consistent with the broader $T_{\mathrm{i}}$ and $\omega_{\phi}$ profiles and a suppression of anomalous ion thermal and momentum transport over a wider region of the plasma with counter-NBI heating.

\section{Simulations}

It is well known that radially sheared equilibrium flows $V$ can suppress turbulence if the $E \times B$ shearing rate $\gamma_{E}$ is 

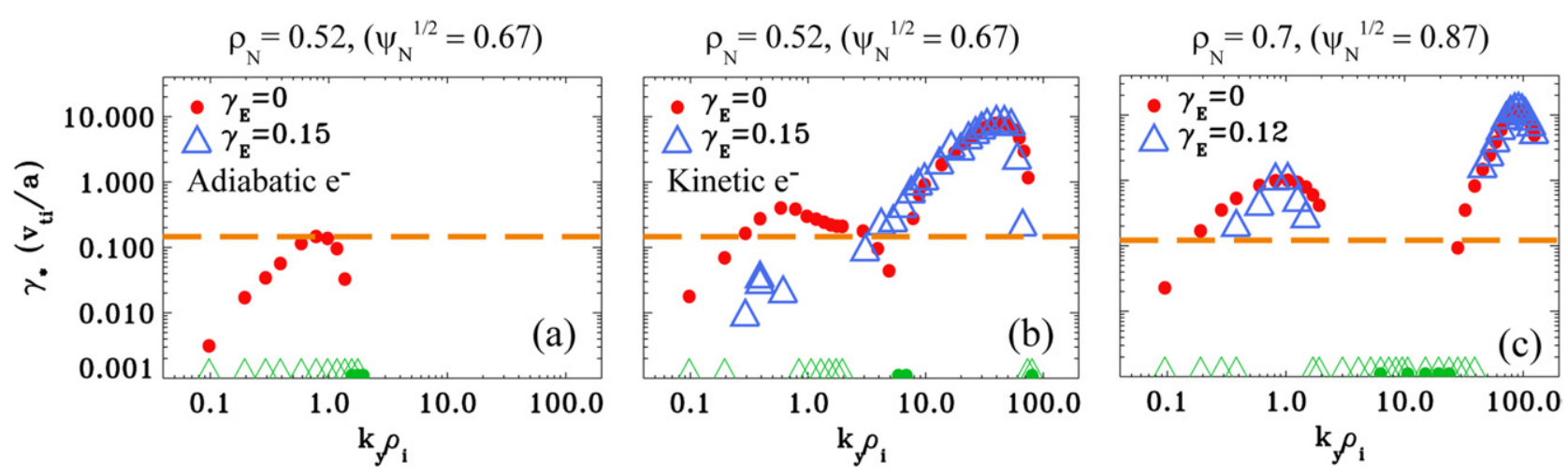

Figure 10. Effective linear growth rate $\gamma_{*}$ versus perpendicular wave-number $k_{y} \rho_{\mathrm{i}}$ with sheared equilibrium toroidal flow (triangles) and without sheared flow (red dots) for co-NBI ITB discharge \#22807 (0.25 s) at $(a)$, $(b)$ mid-radius and $(c)$ in the outer region. Simulations were carried out with: $(a)$ kinetic ions and adiabatic electrons and $(b)$, $(c)$ with kinetic electrons and ions. The horizontal dashed lines show the local level of flow shear $\gamma_{E}$ and the green triangles at the axis minimum denote stable modes.

larger than the maximum linear growth rate $\gamma_{\max }$ [25], i.e. $\gamma_{E}=\mathrm{d} V_{\perp} / \mathrm{d} r>\gamma_{\max }$. ITG driven turbulence can be affected when $\gamma_{E} \sim \mathrm{O}\left(v_{\mathrm{th}, \mathrm{i}} / L\right)$, where $L$ is an equilibrium scale length, e.g. the ion temperature scale length $L_{\mathrm{T}}$. Equilibrium flows which approach sonic speeds are predominantly toroidal as the poloidal component generated by $E_{r}$ is largely cancelled by the parallel neo-classical flow. Equilibrium diamagnetic flows have a poloidal component but these smaller flows $v_{\mathrm{d}} \sim\left(\rho_{\mathrm{i}} / L\right) v_{\mathrm{th}, \mathrm{i}}$ can only influence ITG modes if they vary over the $\rho_{\mathrm{i}}$ length scale which violates the gyro-kinetic ordering assumptions. Sheared toroidal equilibrium flow $V_{\phi}=R \omega(\psi) e_{\phi}$ has been implemented in the local, flux-tube geometry, gyro-kinetic code GS2 [26] in the intermediate flow ordering, where $v_{\text {th,i }} \gg V \gg\left(\rho_{\mathrm{i}} / L\right) v_{\text {th,i }}$ [7]. Sheared flows introduce two additional physics terms into the gyro-kinetic equation. The perpendicular component $\gamma_{E}$ tears apart radially extended eddies and is generally stabilizing, while the parallel component $\sim\left(B_{\phi} / B_{\theta}\right) \gamma_{E}$ drives the parallel velocity gradient (PVG) driven instability [27] and can enhance the growth rate of ITG modes [28]. The ratio of turbulence suppression to additional drive is proportional to $\left(B_{\theta} / B_{\phi}\right) \sim r /(R q)$, i.e. suppression is favoured at low $q$ and at large $r / R$, which arises by definition in a ST.

Non-linear simulations for the conventional aspect ratio Cyclone base case equilibrium have demonstrated that the sheared parallel component of the flow can re-kindle turbulence at sufficiently large sheared flow [7,29,30]. This analysis has recently been extended to study turbulent toroidal momentum transport, and to assess the detailed sensitivity of turbulent fluxes to $\rho_{\mathrm{s}} / L_{T_{\mathrm{i}}}$ and $\gamma_{E}[31,32]$. At high values of sheared flow or low magnetic shear, the sheared parallel flow transiently drives instability growth, which can support sub-critical turbulence observed in recent simulations [31-33]. Sub-critical turbulence is characterized by transient growth of instabilities in non-linear simulations under conditions where there are no linearly unstable modes. Furthermore, a possible physics mechanism, through which plasma equilibria may bifurcate to ITB profiles with steeper gradients, has been found to be more effective when the magnetic shear is weak or negative [31]. These results may be relevant to ITB formation in MAST.

A procedure to determine effective linear growth rates in local linear gyro-kinetic simulations with flow shear was outlined in [7]. In previous work [7,34], local linear electrostatic analyses were performed at close to mid-radius $\left(\rho_{\mathrm{n}}\left(=\Phi_{\mathrm{N}}^{1 / 2}\right)=0.4\right.$, where $\Phi_{\mathrm{N}}$ is the normalized toroidal flux $)$ for the MAST H-mode plasma \#6252. The trapped particle drives for low- $k$ modes $\left(k_{y} \rho_{\mathrm{i}}<\mathrm{O}(1)\right)$ were shown to be weak owing to collisions, and the flow shear was found to be sufficient to stabilize all linear, low- $k$ electrostatic instabilities in this region [7].

A similar linear analysis has been performed for the co-NBI ITB discharge \#22807 (which is similar to \#24600 shown in figure 3$)$ at the time of peak ITB strength $(0.25 \mathrm{~s})$ at three radial locations of $\rho_{\mathrm{N}}=0.3,0.52$ and 0.7 , which are located inside the ITB region, just outside $q_{\min }$ and in the outer region of the plasma. At the innermost location where the magnetic shear is negative, the linear electrostatic analysis shows that all modes at both ion and electron scales are stable with or without flow shear. Including magnetic perturbations without sheared flow did not change this result. (There is no plot associated with this surface shown in figure 10.)

As shown in figures $10(a)$ and $(b)$, at mid-radius, just outside $q_{\text {min }}$ where $\hat{s}$ is slightly positive, it is important to include kinetic electron physics because there is an appreciable trapped electron drive for TEM modes $\left(k_{y} \rho_{\mathrm{i}}<\mathrm{O}(1)\right)$ at that surface. It is important to note that the collisionality is lower and the density gradient higher than in the H-mode equilibrium discussed in [7,34], hence, with kinetic electrons unstable TEM modes appear in the intermediate $k$ region $\left(1<k_{y} \rho_{\mathrm{i}}<10\right)$ between the ITG and ETG scales. Including flow shear and kinetic electrons results in substantial but incomplete suppression of these instabilities (see figure $10(b)$, blue triangles). (In contrast, with adiabatic electrons ITG modes are unstable without flow shear (red dots) but including the modest level of flow shear completely stabilizes these modes (see figure 10(a)).)

On the outermost surface, a stable gap appears in the spectral range between the ITG and ETG scales: i.e. the TEM modes are stable because the plasma is more collisional. Strongly growing ITG modes $\left(k_{y} \rho_{\mathrm{i}}<\mathrm{O}(1)\right)$ are not fully stabilized by the weak flow shear in this outer region. Anomalous thermal and momentum transport is hence to be expected in this region, which is consistent with observations (see figure 4(c)). Doubling the flow shear would be sufficient 


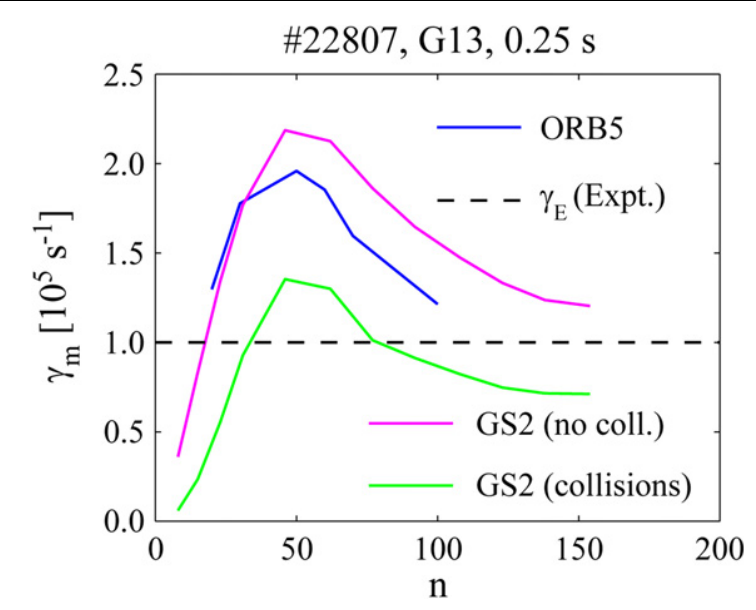

Figure 11. Growth rates (TEM) from linear GS2 (local, with/without collisions) and ORB5 (global) calculations with kinetic electrons. The local $E \times B$ shearing rate is indicated.

to fully suppress the ITG modes here. In summary, these calculations indicate that flow shear is not required to sustain the ITB in the core region with negative magnetic shear but that it is acting to reduce the level of transport due to low- $k$ TEM modes at mid-radius. The outer region is, however, unstable to ITG modes at the prevailing level of flow shear, which would result in anomalous ion heat transport consistent with observations.

Note that the electron-scale (ETG) turbulence does not result in any ion thermal transport and can only produce transport at the experimentally observed levels in the electron channel through the development of radially extended 'streamer' structures in the non-linearly saturated turbulence [17]. Stochasticity due to overlapping magnetic islands from micro-tearing instabilities is another possible explanation of the anomalous electron transport in these plasmas [34]. A full discussion of the anomalous electron transport is beyond the scope of this paper.

The linear stability to ITG modes of discharge \#22807 at $0.25 \mathrm{~s}$ when the ITB is strongest has been analysed using the electrostatic, global gyro-kinetic code ORB5 $[8,35]$ and the GYRO [36] code in local mode at the most unstable location $\left(\psi_{\mathrm{N}}^{1 / 2}=0.67\right)$ and the growth rate spectra from the two codes are found to be in good agreement. With adiabatic electrons, the maximum growth rates for ITG modes $\gamma_{\text {ITG }}$ are found to be below the experimental shearing rate $\gamma_{E}$ by a factor of $\sim 2$. Recent modifications to ORB5 incorporate the equilibrium flow shear self-consistently. Subsequent linear runs with flow shear indicate that this level of flow shear $\left(\gamma_{E} / \gamma_{\mathrm{ITG}} \sim 2\right)$ is sufficient to stabilize the ITG modes completely at this surface.

With kinetic electrons, higher linear growth rates $\gamma_{\text {TEM }}$ are found both with ORB5 and GS2, which exceed the shearing rate by a factor of $\sim 2$, as shown in figure 11 , although including collisions in the GS2 calculations slightly reduces the growth rates. The linear analysis also shows that the core region $\left(\psi_{\mathrm{N}}^{1 / 2}<0.6\right)$ is stable, while unstable region is localized in the outer part of the plasma where the magnetic shear is positive. These results are consistent with the GS2 calculations discussed above.

In the event where low- $k$, ion-scale micro-instabilities are present, global, non-linear simulations are required to

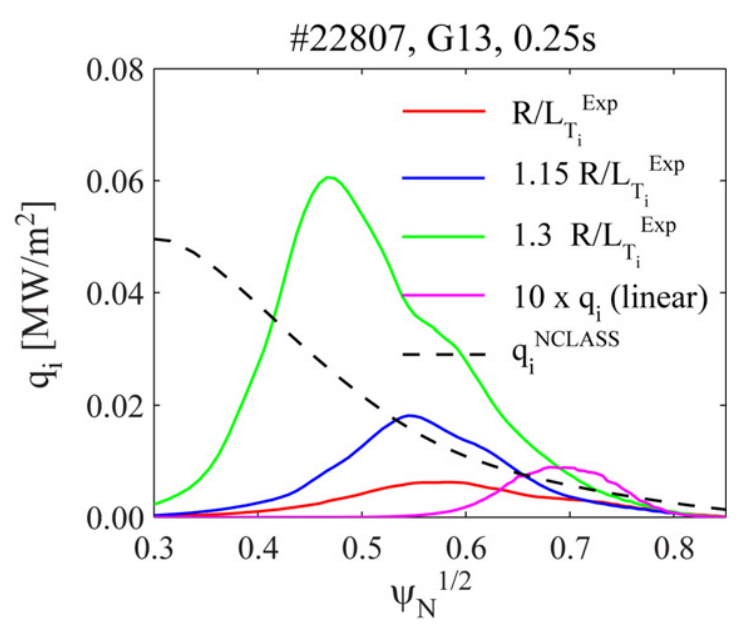

Figure 12. Ion heat flux $q_{\mathrm{i}}$ from non-linear ORB5 calculations with adiabatic electrons for cases with $R / L_{T_{\mathrm{i}}}$ at the experimental value and increased by a factor of 1.15 and 1.3 . The ion heat flux from NCLASS and the linear flux are also shown.

capture equilibrium variation over the large domain required for the simulation. This has motivated modelling of the ITG turbulence in this ITB discharge using ORB5 [8]. Simplified, non-linear ITG simulations without flow shear and with adiabatic electrons show that the turbulence spreads inwards from the linearly unstable outer region to the stable inner region. With the experimental $T_{\mathrm{i}}$ gradient, the ion heat flux $q_{\mathrm{i}}$ saturates at just below the ion neo-classical level. As shown in figure 12 , modest increases $(30 \%)$ in the gradient result in considerably increased ion thermal transport indicating that the ITG turbulence may be clamping the experimental $T_{\mathrm{i}}$ profiles, i.e. that the ion transport is stiff in the region outside the ITB. Global, non-linear simulations with both sheared equilibrium flow and kinetic electrons are clearly required to capture fully the dynamics of the ion scale turbulence.

\section{Summary and conclusions}

ITBs in the ion thermal and momentum channels form in MAST L-mode discharges with negative magnetic shear in the vicinity of $q_{\mathrm{min}}$. With co-NBI heating, an ITB in the momentum channel forms first in a region of negative magnetic shear in the core plasma. Some correlation is found with the strength of the ITB and the passing of $q_{\text {min }}$ through rational values. An ITB also forms in the ion thermal channel at somewhat larger radius, where the ion thermal transport is at the neo-classical level, resulting in $T_{\mathrm{i}}$ exceeding $T_{\mathrm{e}}$ in the plasma core. The strength of the ITB is limited by coupling of MHD modes which reduce the rotation gradient and ultimately, an internal kink mode removes the core flow shear destroying the ITB when $q_{0}$ approaches unity. Micro-stability analysis indicates that the negative magnetic shear core region is stable, and flow shear is not required to form the ITB there, while in the outer regions the flow shear is too weak to fully stabilize low- $k$ ITG modes resulting in anomalous ion transport. At mid-radius shear flow is sufficient to stabilize ITG modes; however, weakly growing low- $k$ TEM modes remain over a narrower range of wave numbers. The conclusion that the negative shear region is stable and does not require shear-flow 
stabilization for ITB formation is consistent with findings of dimensionless identity experiments on ITB formation on JET and JT-60U [37]. In the positive shear region the electronscale (ETG) modes are not stabilized by the flow shear. A discussion of the electron thermal transport in these discharges is, however, beyond the scope of this paper.

With counter-NBI, the absorbed power is considerably reduced due to increased prompt fast-ion losses, although the torque is comparable to that with co-NBI heating. The confinement is improved compared with that with co-NBI, particularly of particles and momentum. The rotation profile is broader than with co-NBI, with the momentum diffusivity below the ion thermal neo-classical level across a broader region of the plasma. Due to the higher density and lower temperature, the ion and electron temperatures are similar because of increased ion-electron exchange. Although there is no distinct ITB observable as with co-NBI, where $T_{\mathrm{i}}$ exceeds $T_{\mathrm{e}}$ in the core, there is an observable maximum in the normalized $T_{\mathrm{i}}$ gradient, which is initially localized just outside $q_{\min }$. This later bifurcates with an inner maxima close to $q_{\min }$ and an outer maxima which tracks $q=5 / 2$ as it evolves outwards. The ion thermal diffusivity is also close to the neo-classical level in the core plasma, with this region of suppressed anomalous transport broadening as the discharge evolves.

Measurements of the low- $k$ density turbulence in such plasmas using a BES imaging system newly installed on MAST [38] will in future allow direct comparison of results from global turbulence simulations with observations.

\section{Acknowledgments}

This work was funded by the RCUK Energy Programme under grant EP/G003955 and the European Communities under the contract of Association between EURATOM and CCFE. The views and opinions expressed herein do not necessarily reflect those of the European Commission.

\section{(C) 2011 Euratom.}

\section{References}

[1] Akers R.J. et al 2003 Plasma Phys. Control. Fusion 45 A $175-204$

[2] Field A.R., Akers R.J., Brickley C., Carolan P.G., Challis C., Conway N.J., Cunningham G, Meyer H, Roach C., Walsh M.J. and the MAST Team 2004 Core heat transport in the MAST spherical tokamak Proc. 20th Fusion Energy Conf. (Vilamoura, Portugal, 2004) (Vienna: IAEA) EX/P2-11 http://www-naweb.iaea.org/napc/physics/fec/fec2004/ datasets/EX_P2-11.html

[3] Scannell R. et al 2008 Rev. Sci. Instrum. 79 10E730

[4] Conway N.J. et al 2006 Rev. Sci. Instrum. 77 10F131

[5] Conway N.J., DeBock M.F.M., Michael C.A., Walsh, M.J., Carolan P.G., Hawkes N.J., Rachlew E., McCone J.F.G., Shibaev S. and Wearing G. 2010 Rev. Sci. Instrum. 81 10D738

[6] Hawryluk R.J. 1980 An empirical approach to tokamak transport Physics of Plasmas Close to Thermonuclear Conditions ed B. Coppi et al (Brussels: CEC) vol 1, pp 19-46 http://w3.pppl.gov/transp/papers/Hawryluk.pdf

[7] Roach C.M. et al 2009 Plasma Phys. Control. Fusion 51124020
[8] Saarelma S., Bottino A., Candy J., Colyer G., Field A.R., Gutenfelder W., Hill P., Peeters A., Roach C.M. and the MAST team 2010 Global gyrokinetic ITG turbulence simulations of MAST plasmas 37th EPS Conf. on Plasma Physics Controlled Fusion (Dublin, Ireland, 2010) P1-1085 http://ocs.ciemat.es/EPS2010PAP/pdf/P1.1085.pdf

[9] Connor J.W., Fukuda T., Garbet X., Gormezano C., Mukhovatov V., Wakatani M., the ITB Database Group and the ITPA Topical Group on Transport and Internal Barrier Physics 2004 Nucl. Fusion 44 R1-R49

[10] Wolf R.C. 2003 Plasma Phys. Control. Fusion 45 R1-91

[11] Joffrin E. et al 2002 Plasma Phys. Control. Fusion 441739

[12] Günter S. et al 2001 MHD phenomena as trigger for the formation of internal transport barriers on ASDEX upgrade28th EPS Conf. on Plasma Physics (Funchal, Portugal, 2001) vol 25A (Lisbon: European Physical Society) p 49 http://epsppd.epfl.ch/Madeira/html/ authors/nav/AutG04fr.html

[13] Garbet X., Bourdelle C., Hoang G.T., Maget P., Benkadda S., Beyer P., Figarella C., Voitsekovitch I., Agullo O. and Bian N. 2001 Phys. Plasmas 82793

[14] Romenelli F. and Zonca F. 1993 Phys. Fluids B 54081

[15] Drake J.F., Lau Y.T., Guzdar P.N., Hassam A.B., Novakovski S.V., Rogers B. and Zeiler A. 1996 Phys. Rev. Lett. 77494

[16] Beer M.A., Hammett G.W., Rewoldt G., Synakowski E.J., Zarnstorff M.C. and Dorland W. 1997 Phys. Plasmas 41792

[17] Austin M.E. et al 2006 Phys. Plasmas 13082502

[18] Yuh H.Y. et al 2009 Phys. Plasmas 16056120

[19] Field A.R., Akers R.J., Brickley C., Carolan P.G., Conway N.J., Cunningham G., Meyer H., Walsh M.J., Challis C. and the MAST Team 2004 Transport in the presence of ITBs in the MAST Spherical Tokamak 31st EPS Conf. on Plasma Physics and Controlled Fusion (London, 2004) vol 28G (ECA) P4-187, ISBN 2-914771-22-3 http://epsppd.epfl.ch/London/pdf/P4_187.pdf

[20] Michael C. et al 2010 Internal transport barriers in the MAST spherical tokamak 37th EPS Conf. on Plasma Physics and Controlled Fusion (Dublin, Ireland 2010) vol 34A (ECA) P1.1067, ISBN 2-914771-62-2 and http://ocs.ciemat.es/EPS2010PAP/html/

[21] Hua M.-D., Chapman I.T., Field A.R., Hastie R.J., Pinches S.D. and the MAST Team 2009 Plasma Phys. Control. Fusion 52035009

[22] Fitzpatrick R. 1995 Phys. Plasmas 2825

[23] Chapman I.T., Hua M.-D., Pinches S.D., Akers R.J., Field A.R., Graves J.P., Hastie R.J., Michael C.A. and the MAST Team 2010 Nucl. Fusion 50045007

[24] Connor J.W., Cowley S.C., Hastie R.J. and Pan L.R. 1987 Plasma Phys. Control. Fusion 29 919-31

[25] Waltz R.E., Kerbel G.D. and Milovich J. 1994 Phys. Plasmas 12229

[26] Kotschenreuther M., Rewoldt G. and Tang W.M. 1995 Comput. Phys. Commun. 88128

[27] Catto P.J., Rosenbluth M.N. and Liu C.S. 1973 Phys. Fluids 161719

[28] Peeters A.G. and Angioni C. 2005 Phys. Plasmas 12072515

[29] Dimits A.M. et al 2000 Phys Plasmas 7969

[30] Kinsey J., Waltz R.E. and Candy J.M. 2005 Phys. Plasmas 12062302

[31] Barnes M., Parra F.I., Highcock E.G., Schekochihin A.A., Cowley S.C. and Roach C.M. 2010 Phys. Rev. Lett. submitted and http://arxiv.org/abs/1007.3390

[32] Highcock E.G., Barnes M., Schekochihin A.A., Parra F.I., Roach C.M. and Cowley S.C. 2010 Phys. Rev. Lett. 105215003

[33] Newton S.L., Cowley S.C. and Loureiro N.F. 2010 Plasma Phys. Control. Fusion 52125001

[34] Applegate D.J. et al 2004 Phys. Plasmas 115085

[35] Jolliet S., Bottino A., Angelino P., Hatzky R., Tran T.M., Mcmillan B.F., Sauter O., Appert K., Idomura Y. 
and Villard L. 2008 Comput. Phys. Commun. 177 409-25

[36] Waltz R.E., Candy J.M. and Rosenbluth M.N. 2002 Phys. Plasmas 91938

[37] Litaudon X. et al 2010 Core transport properties in JET and JT-60U identity plasmas 23rd IAEA Fusion Energy Conf.
(Daejeon, Korea, 11-16 October 2010) EXC-P4-12

http://www-pub.iaea.org/MTCD/Meetings/PDFplus/2010/ cn180/cn180_papers/exc_p4-12.pdf

[38] Field A.R., Dunai D., Conway N.J., Zoletnik S. and Sárközi J. 2009 Rev. Sci. Instrum. 80073503 